\title{
In-flight calibration of SCIAMACHY's polarization sensitivity
}

\author{
Patricia Liebing $^{1, \mathrm{a}}$, Matthijs Krijger ${ }^{2, \mathrm{~b}}$, Ralph Snel ${ }^{2, \mathrm{c}}$, Klaus Bramstedt ${ }^{1}$, Stefan Noël ${ }^{1}$, Heinrich Bovensmann ${ }^{1}$, and \\ John P. Burrows ${ }^{1}$ \\ ${ }^{1}$ Institute of Environmental Physics (IUP), University of Bremen, Bremen, Germany \\ ${ }^{2}$ SRON Netherlands Institute for Space Research, Sorbonnelaan 2, 3584 CA Utrecht, the Netherlands \\ ${ }^{a}$ now at: Leiden Observatory, 2300 RA Leiden, the Netherlands \\ ${ }^{b}$ now at: Earth Space Solutions, Utrecht, the Netherlands \\ ${ }^{c}$ currently at: TNO, Stieltjesweg 1, 2628 CK Delft, the Netherlands
}

Correspondence: Patricia Liebing (liebing@strw.leidenuniv.nl)

Received: 1 June 2017 - Discussion started: 31 July 2017

Revised: 3 November 2017 - Accepted: 20 November 2017 - Published: 15 January 2018

\begin{abstract}
This paper describes the in-flight calibration of the polarization response of the SCIAMACHY polarization measurement devices (PMDs) and a selected region of its science channels. With the lack of polarized calibration sources it is not possible to obtain such a calibration from dedicated calibration measurements. Instead, the earthshine itself, together with a simplified radiative transfer model (RTM), is used to derive time-dependent and measurementconfiguration-dependent polarization sensitivities. The results are compared to an instrument model that describes the degradation of the instrument as a result of a slow buildup of contaminant layers on its elevation and azimuth scan mirrors. This comparison reveals significant differences between the model prediction and the data, suggesting an unforeseen change between on-ground and in-flight calibration in at least one of the polarization-sensitive components of the optical bench. The possibility of mechanisms other than scan mirror contamination contributing to the degradation of the instrument will be discussed. The data are consistent with a polarization phase shift occurring in the beam split prism used to divert the light coming from the telescope to the different channels and polarization measurement devices. The extension of the instrument degradation model with a linear retarder enables the determination of the relevant parameters to describe this phase shift and ultimately results in a significant improvement of the polarization measurements as well as the polarization response correction of measured radiances.
\end{abstract}

\section{Introduction}

SCIAMACHY (Bovensmann et al., 1999) was a passive spectrometer onboard ESA's Envisat platform, operational between 2002 and 2012. Spectra of the radiance upwelling at the top of the atmosphere in nadir, limb and occultation geometries as well as of the extraterrestrial solar irradiance were measured by SCIAMACHY. The uncalibrated level 0 data are transformed by applying the instrument response functions, obtained during preflight and in-flight calibration, to the radiance, irradiance and reflectance. Measurements between 214 and $1750 \mathrm{~nm}$ were contiguously recorded in six channels at wavelength-dependent spectral resolutions between 0.2 and $1.5 \mathrm{~nm}$. In addition, there were two channels with bands around 2.0 and $2.3 \mu \mathrm{m}$ and spectral resolutions around $0.2 \mathrm{~nm}$ and seven polarization measurement devices, PMDs. The latter were broadband detectors at selected wavelengths. Combined with the spectral channels they were used to determine the polarization of the upwelling radiation. The resulting radiance, irradiance and reflectance data products were used to derive column amounts and concentrations of atmospheric trace gases and aerosol and cloud data products. The accuracy of these data products depends critically on that of the measured spectra, i.e., the absolute magnitude of the radiometric signals and their spectral shape. Therefore, it is vital to obtain a highly accurate characterization of the instrument response functions to unpolarized and polarized light, both from on-ground measurements and in-flight measurements. SCIAMACHY is equipped with scan mirrors to reflect the light from nadir or limb line of sights into the 
telescope and then further towards grating spectrometers and photodiode detectors. The degradation of the scan mirrors can be modeled with a thin, slowly growing layer of contaminant on top of their oxidized aluminum surfaces. In-flight calibration measurements for SCIAMACHY were carried out regularly with unpolarized sources, mainly the Sun, and for different scan mirror configurations and positions. These data have been analyzed with the so-called scan mirror model which, by applying the Fresnel equations for layered surfaces to these specific measurement configurations, determined the refractive indices and thicknesses of the contaminant on each of the two scan mirrors (Krijger et al., 2014). Indeed, a major part of the observed instrumental throughput degradation in the UV, and its scan angle dependence, can be explained by the contamination of the scan mirrors. The remaining part is attributed to the degradation of the optical bench module (OBM), which comprises all elements of the optical train behind the instrument slit.

The spectrometer grating and also the scan mirrors and other optical components along the light path are polarization sensitive, meaning that their optical properties depend partially on the polarization of the incident light. The polarization sensitivity of the instrument as a whole has been measured on the ground (Gottwald and Bovensmann, 2011) and found to be substantial, on the order of $20 \%$ or more, over large wavelength ranges. The measured polarization values, obtained from the combination of PMD and science channel measurements, can be used to correct the signal of the SCIAMACHY science channels. For this correction to be effective, both the polarization and the polarization response have to be known with sufficient accuracy. Measured polarization and reflectance values in the visible and nearinfrared range have been compared to POLDER data and found to be within the expected range for selected geometries in nadir (Tilstra and Stammes, 2007, 2010). Limb polarization data on the other hand differed systematically from radiative transfer model (RTM) results (Liebing et al., 2013), an effect which is largest in the UV and decreases towards longer wavelengths. Of importance is the observation that, even though the data show a significant drift over time, a difference between in-flight and preflight measurements is already present early in the mission. These findings imply that there were either unidentified systematic errors in the onground calibration or perhaps a sudden change in the polarization calibration parameters shortly after launch, followed by an in-flight degradation of polarization-sensitive components. Due to the variation in the observation geometry - and therefore in the polarization - with latitude and season, errors in the polarization determination or correction may lead to latitudinally and seasonally dependent systematic errors in the derived atmospheric trace gas concentrations. Moreover, long-term degradation effects may consequently lead to drifts in the data if not appropriately accounted for.

Recently, the on-ground calibration data have been reanalyzed, making use of the scan mirror model for SCIA-
MACHY (Krijger et al., 2014). The application of this model for uncontaminated mirrors during the on-ground calibration campaign enables the separation of the radiance and polarization sensitivities of the scan mirrors from that of the OBM. The knowledge of the OBM polarization response can then be combined with that on the mirror contamination in space derived from the in-flight calibration measurements. The application of the model to nadir and limb measurement configurations yields a prediction of the time-dependent behavior of in-flight polarization sensitivities for limb and nadir observations.

The aim of this paper is to devise a method for the direct in-flight calibration of the polarization response which enables a validation of the scan mirror model and its derived parameters as well as a correction for the suspected on-ground to in-flight change. Furthermore, an investigation of this onground to in-flight change leads to its most likely culprit: a polarization phase shift induced in the beam split prism that is used to separate the almost completely polarized beams for the PMDs from the partially polarized beams for the science detectors. Such a phase shift can be modeled with an extension to the scan mirror model that includes a linear retarder and thus modifies the OBM polarization sensitivities as measured on the ground.

A polarized calibration source is necessary in order to derive the in-flight polarization sensitivities. As the Sun and onboard calibration sources are unpolarized, the only available polarized calibration source is the earthshine itself. By exploiting the relationship between depolarization and intensity at fixed wavelength and viewing geometry, data points with known polarization can be selected and sampled in order to obtain the polarization sensitivities. The information available in the data permits this to be done only for the PMDs and for a particular feature in SCIAMACHY's science channel at around $350 \mathrm{~nm}$. The statistical nature of this approach mitigates errors arising from the underlying assumptions to a certain degree; the remaining errors are to be estimated from the systematic variation of those assumptions. The results obtained in the UV and, partially, visible (VIS) regions prove to be accurate enough to deduce from them the parameters of the instrumental phase shift and its possible cause. This investigation may also be relevant in view of other instruments whose design follows a similar principle, such as the GOME series on the ERS-2 and MetOp satellites.

The structure of this article is as follows: in Sect. 2, first the polarization formalism used here is introduced, including the Mueller matrix approach and the description of the polarization coordinate frame. Next, the SCIAMACHY polarization measurements are explained in detail. Section 3 explains the calibration strategy and presents the results. The interpretation of the results in terms of an on-ground to in-flight change in the OBM and possibly its further degradation is presented in Sect. 4. This section also contains a discussion on implications regarding the status of the instrument before and af- 
ter launch and on the applicability of the scan mirror model. Conclusions are presented in Sect. 5.

\section{Polarization formalism and measurements}

Throughout this paper, we employ the Mueller matrix formalism to describe the instruments sensitivity to polarized light. The formalism, frame definitions and application to SCIAMACHY are presented in this section together with a description of the polarization measurement approach.

\subsection{Mueller matrix formalism and polarization frame}

Formally, the measured signal of any given polarizationsensitive instrument is the first element of the Stokes vector resulting from the product of the incoming Stokes vector $\boldsymbol{I}=(I, Q, U, V)^{T}$ with the $4 \times 4$ Mueller matrix that describes its modification by the optical elements inside the instrument:

$\boldsymbol{S}_{0}=[\mathbf{M} \cdot \boldsymbol{I}]_{0}$.

The Stokes vector components are combinations of the intensities $I$ with different polarization planes with respect to a given reference frame:

$$
\begin{aligned}
& I=I_{\|}+I_{\perp}, \quad Q=I_{\|}-I_{\perp}, \quad U=I_{45^{\circ}}-I_{-45^{\circ}}, \\
& V=I_{R}-I_{L} .
\end{aligned}
$$

The linear polarization is defined by the $Q$ and $U$ components; $V$ is the circular polarization component. Often, the Stokes vector is given in relative terms, i.e., $I=$ $I(1, q, u, v)^{T}$, where the second to fourth components can take values between -1 and 1 and the total degree of polarization is defined as

$p \equiv \sqrt{q^{2}+u^{2}+v^{2}} \leq 1$.

The Stokes vector frame is defined by choosing the parallel $(\|)$ axis. The perpendicular $(\perp)$ axis is then naturally given, while for the $\pm 45^{\circ}$ direction as well as the left $(L)$ and right $(R)$ handedness of the circular polarization a sense of orientation needs to be defined. Here, the sense of orientation is given by looking into the travel direction of the light, i.e., towards the instrument. A positive rotation is defined to be clockwise, i.e., left-handed.

The elements of the Mueller matrix can be interpreted in the following way: $M_{11}$ is the optical throughput for unpolarized light, i.e., $\boldsymbol{I}=(I, 0,0,0)^{T}$. The other diagonal elements $M_{i i}$ describe the transfer efficiency for a given input Stokes vector component ( $Q$ for $i=2, U$ for $i=3, V$ for $i=4$ ) to the output Stokes vector, while the non-diagonal elements stand for the crosstalk between different Stokes vector components. The instrument Mueller matrix itself is the product of the Mueller matrices of its individual components. The
Mueller matrix needs to be given in the same reference frame as the Stokes vector and, if necessary, reference frames for individual components have to be transformed to this particular reference frame.

Since only the first component of the signal Stokes vector $S_{0} \equiv S$ is being measured, only the first row of the instrument Mueller matrix is needed for calibration of the detector signal:

$S=I M_{11}\left(1, \mu_{2}, \mu_{3}, \mu_{4}\right)(1, q, u, v)^{T}$,

where the $\mu_{i}=M_{1 i} / M_{11}$ are the normalized Mueller matrix elements (MMEs). Note that, if the end-to-end Mueller matrix is derived from a combination of individual matrices, all but the last one of those still have to be known completely.

For SCIAMACHY, the Stokes frame for the measured atmospheric polarization is defined with respect to the local meridional plane, the plane spanned by the line of sight and the local zenith. Parallel polarization, i.e., $q=+1$, is given when the polarization lies in this plane. Positive $45^{\circ}$ polarization $u=+1$ is defined for the polarization plane which is rotated clockwise by $45^{\circ}$ from the parallel direction, when looking along the line of sight into the instrument. For calibration purposes and within the scan mirror model, another frame definition is applied. The end-to-end Mueller matrix or Stokes vectors can easily be transformed between these different frames. In this paper we work entirely with the atmospheric polarization, and, to avoid confusion, we choose to give the end-to-end Mueller vector in the atmospheric polarization frame.

\subsection{The scan mirror model}

The details of the scan mirror model and its application to SCIAMACHY are described in Krijger et al. (2014). Essentially, the instrument is separated into a scan module consisting of one or several scan mirrors and the optical bench module which comprises everything else, i.e.,

$S=I M_{11}^{\mathrm{OBM}} \boldsymbol{\mu}^{\mathrm{OBM}} \mathbf{M}(\alpha)(1, q, u, v)^{T}$.

Here, $\boldsymbol{\mu}^{\mathrm{OBM}}$ is the first row vector of the normalized OBM Mueller matrix, $M_{11}^{\mathrm{OBM}}$ is the unpolarized radiance response of the OBM and $\mathbf{M}(\alpha)$ is the scan mirror Mueller matrix which depends on the incidence angle(s) $\alpha$. Depending on the scan mirror configuration in use (one or more mirrors with reflection planes at an angle), it can be the product of several Mueller matrices appropriately transformed to match their respective coordinate systems. The Mueller matrix of any of the scan mirrors can be calculated from the Fresnel equations, possibly taking into account multiple layers of material and knowing their complex refractive indices and thicknesses. The separation of the instrument into these two modules enables two separate calibration steps: on the ground, the calibration data which were obtained with particular scan mirror configurations can be modeled with uncontaminated 
mirrors to yield the OBM Mueller vector (Krijger, 2017). In-flight, the calibration measurements with a stable, unpolarized source (the Sun or the internal white light source) can be used to determine the thickness and refractive index of a contaminant layer on each of the involved scan mirrors, assuming a fixed OBM. Realistically, the limited information contained in the combination of calibration measurements used only allows for the determination of a single wavelength-dependent refractive index which is constant in time and the time-dependent thicknesses on each of the scan mirrors. Additionally, an extra factor, called "M-factor", is introduced to indicate the degradation of the unpolarized OBM response. However, the separation of mirror and OBM degradation given the information contained in the calibration measurements is not entirely unambiguous - in the sense that to some degree both can describe the unpolarized calibration measurements equally well.

\subsection{Nadir and limb scans}

The relevant measurements discussed here are nadir and limb reflectance and polarization measurements. SCIAMACHY performs both observation modes in an alternating sequence. Envisat's Sun-synchronous orbit crosses the descending node at 10:00 local time, such that the Sun is always to the left of the flight direction, with a minimum solar zenith angle (SZA) of about $20^{\circ}$.

A nadir scan is carried out by pointing the ESM (elevation scan module) mirror toward the surface and then continuously scanning from left to right, thereby covering elevation angles up to $32^{\circ}$ and a total swath width of $960 \mathrm{~km}$. During the scan, the detector signal is read out several times. The integration time depends on wavelength and SZA. The data considered here are all selected to have integration times of $0.25 \mathrm{~s}$ or less, such that 16 readouts per scan are available. The ground pixel size thus amounts to $60 \mathrm{~km}$ across track as determined by the integration time and $30 \mathrm{~km}$ along track as determined by the length of the instrument slit of $1.8^{\circ}$ and the motion of the spacecraft. The scan takes $4 \mathrm{~s}$ and is followed by a fast backward scan of $1 \mathrm{~s}$. This sequence is typically repeated 13 times before switching to a limb scan.

During a limb scan, the ASM (azimuth scan module) mirror is pointed approximately into the flight direction, toward the limb of Earth's atmosphere. The projection of the instrument slit is parallel to the horizon, providing an instantaneous field of view of about $103 \mathrm{~km}$ horizontally and $2.6 \mathrm{~km}$ vertically. The light is reflected off the ASM mirror toward the ESM mirror which is set at an elevation angle of about $63^{\circ}$. The reflection planes of both mirrors are at an angle to each other. In the scan mirror model, this angle is properly accounted for by rotating the respective coordinate systems. The ASM mirror performs a horizontal scan of about $\pm 10^{\circ}$ around the flight direction at a fixed ESM elevation angle, starting approximately $3 \mathrm{~km}$ below the horizon. The horizontal scan takes $1.5 \mathrm{~s}$, again covering a swath of $960 \mathrm{~km}$. After the scan, the ESM elevation angle is increased by about $3.3 \mathrm{~km}$ and the horizontal scan proceeds in the other direction. Between 29 and 34 such horizontal scans are performed in one sequence, thus covering altitudes between about -3 and $93 \mathrm{~km}$ or higher. After the last scan, another ESM elevation step to an altitude of about $250 \mathrm{~km}$ is executed, and a dark measurement is performed for the last $1.5 \mathrm{~s}$ of the sequence. This dark measurement is typically used to correct the detector signals for potentially orbitphase-dependent dark current conditions. During a horizontal scan, the typical integration time is $0.375 \mathrm{~s}$, resulting in four radiance profiles per limb scan. With Envisat flying at an altitude of about $790 \mathrm{~km}$, the tangent points of a limb scan are about $3300 \mathrm{~km}$ ahead of the subsatellite point. The measurement sequence is designed such that about 7 min after a limb scan a nadir sequence is performed whose ground pixels overlap with the limb tangent points, thus enabling stereoscopic measurements. In Fig. 1, the scan mirror configurations and viewing geometries for the nadir and limb observation modes are depicted on the left and right, respectively. In the nadir configuration, the instrument slit is oriented along the flight direction and therefore perpendicular to the meridional plane, which lies in the scan direction. In limb, the projection of the slit is along the horizon and therefore again perpendicular to the plane connecting the line of sight and local zenith or tangent point. Therefore, in both cases, $q=-1$ when the polarization direction (in the atmospheric Stokes frame) is along the instrument slit projection.

The true light path along the line of sight of a limb measurement depends critically on wavelength and the scene observed. Topography and horizontally inhomogeneous cloud or aerosol layers make observations in the troposphere highly variable. However, in the stratosphere, under relatively stable aerosol conditions, reflectance measurements are quite suitable for systematic studies. A possible issue persists in the contamination with spatial stray light, either from the Sun or from bright scenes below the tangent altitude. The measurements considered here are always done with the relative azimuth angle with the Sun larger than $20^{\circ}$, thereby minimizing the possible contamination with solar stray light. Earthshine stray light, however, is clearly visible at tangent heights (THs) above $40 \mathrm{~km}$ and becomes dominant at altitudes larger than about $60 \mathrm{~km}$. Below $40 \mathrm{~km}$, its contribution is not expected to be larger than a few per cent.

Reflectances are obtained by normalizing limb or nadir radiances with the solar irradiance, which is measured once per day using a combination of a diffuser on the backside of the ESM and the ASM mirror.

\subsection{SCIAMACHY polarization measurements}

Generically, polarization can be measured by modifying the Mueller matrix in Eq. (4) such that the measured signal becomes sensitive to different linear combinations of the Stokes vector components. In order to derive all components unam- 

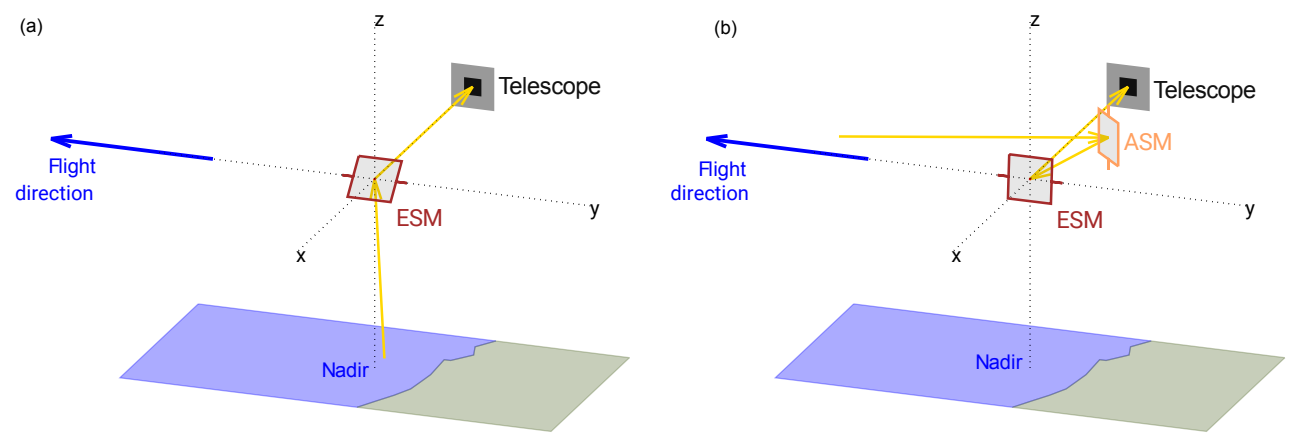

Figure 1. Sketch of the scan mirror configuration and viewing geometry during nadir (a) and limb (b) observations. In nadir mode, the ESM mirror is rotated to achieve a scan across track. In limb mode, the ASM mirror is rotated to achieve a scan across the flight direction, while the ESM mirror performs small steps to select a specific tangent height. Their reflection planes are rotated with respect to each other.

biguously, at least four independent measurements per wavelength have to be carried out. The circular polarization component is typically negligible in atmospheric polarization, reducing the minimum number of required measurements to three. These can be either carried out sequentially, or, as in the case of SCIAMACHY, simultaneously by employing a beam splitter. The beam split device in SCIAMACHY is the so-called "predisperser prism" which is located right after the telescope. It serves two purposes: the first is to spatially separate different spectral ranges of the incoming light in order to facilitate their diversion into SCIAMACHY's eight spectral channels. The second purpose is to split off a small fraction of the beam through internal reflection at the Brewster angle and thus generate an almost fully polarized beam which is directed towards the polarization measurement devices, a set of photodiode detectors sampling at a rate of $40 \mathrm{~Hz}$ and integrating over relatively wide spectral regions (100 to $200 \mathrm{~nm}$ ) that roughly correspond to the central part of each of the main science channels. Figure 2 gives a schematic overview of the measurement principle. After passing the scanner module depicted on the left, the light enters the telescope with the instrument slit. The incidence on the first surface of the predisperser prism is nominal, and thus no spectral diversion occurs inside it. Most of the light exits on the opposite side, which is tilted, and is refracted toward the science channels. The remaining part of the beam undergoes internal reflection under the Brewster angle, after which it is almost fully polarized with its polarization direction pointing into the paper (indicated by the crossed circle), i.e., parallel to the instrument slit. After another internal reflection it exits the prism and is refracted towards the PMDs.

Table 1 lists the PMDs used for this investigation and their relevant properties. The average wavelength given there is approximate, since it depends in detail on the shape of the measured spectrum. Also given in this table are the sensitivity to unpolarized light relative to that of the science channel $\left(\left\langle M_{1}\right\rangle^{\mathrm{PD}}\right)$ and the three components of the OBM Mueller vector, which are all spectrally averaged. PMD 7 is special in the sense that its signal is not split off at the predisperser prism, but rather at the location of channel 5 , and the $45^{\circ}$ polarization is obtained by employing a polarization filter. Its spectral range is nearly identical with that of PMD 4 . While PMD 7 has its largest sensitivity to light polarized at $45^{\circ}$, i.e., $\mu_{3} \approx 1$, the other PMDs are mostly sensitive to light polarized parallel to the direction of the instrument slit; hence $\mu_{2} \approx-1$. Take note of the relatively large sensitivity to circular polarization, which in fact is unexpected given the design of the PMDs and poses a serious problem to the SCIAMACHY polarization measurements. Even though the atmospheric radiance is not circularly polarized, the scan mirrors will convert $45^{\circ}$ linearly polarized light to circularly polarized light. The end-to-end Mueller vector of the PMDs thus contains a large, unwanted sensitivity to $45^{\circ}$ polarization. A similar effect was discovered for the science channels as well. Since it was originally deemed sufficient to determine only $q$ and to correct the science channel radiance for this Stokes component only, a measurement of the $u$ component was not foreseen. This is only possible for the wavelength region around $850 \mathrm{~nm}$, where both PMD 4 and 7 are available. For the other regions, assumptions on the relationship between $q$ and $u$ or on the value of $u$ itself have to be made in order to be able to derive $q$ and to correct the measured signal adequately.

The polarization is determined by equating the synchronized and integrated (over the exposure time of the science channel) PMD signal with the calibrated science channel signal scaled with the PMD response and integrated over the PMD spectral band:

$$
\begin{aligned}
& S^{\mathrm{P}}=\mu_{1}^{\mathrm{PD}} \cdot \sum_{i} S_{i}^{\mathrm{D}} M_{1, i}^{\mathrm{PD}} \frac{1+\mu_{2, i}^{\mathrm{P}} q+\mu_{3, i}^{\mathrm{P}} u}{1+\mu_{2, i}^{\mathrm{D}} q+\mu_{3, i}^{\mathrm{D}} u}, \quad \text { with } \\
& M_{1, i}^{\mathrm{PD}}=\frac{M_{11, i}^{\mathrm{P}}}{M_{11, i}^{\mathrm{D}}} .
\end{aligned}
$$

The sum goes over all pixels in the relevant spectral range; the superscripts $\mathrm{P}$ and D indicate the PMD and science detec- 


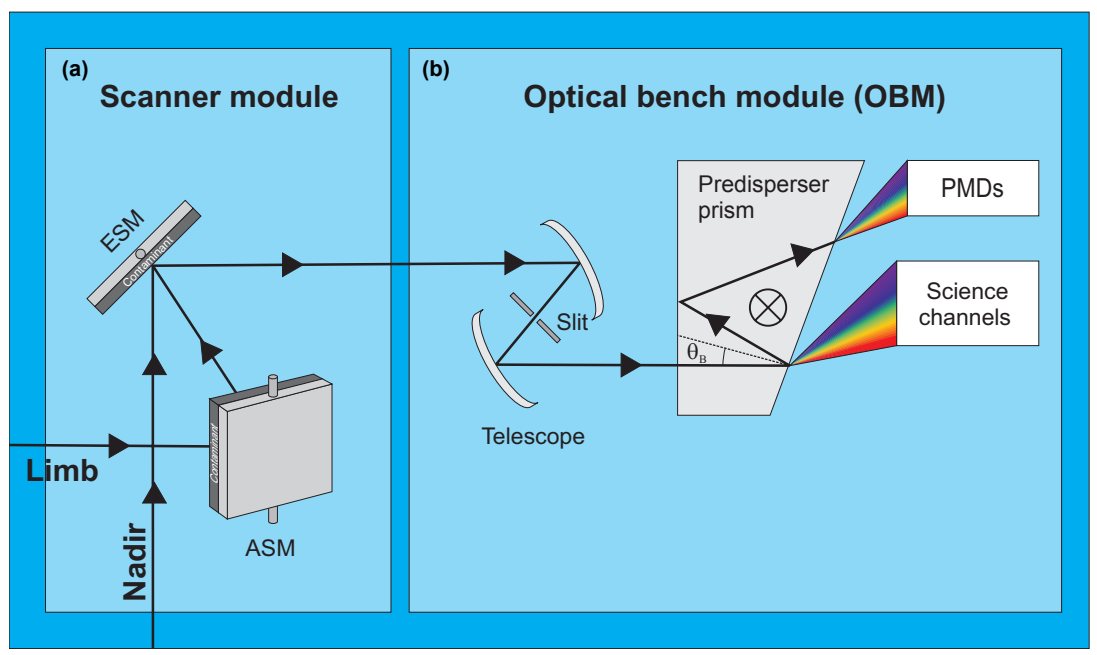

Figure 2. Sketch of the measurement principle of SCIAMACHY. The left part indicates the scanner module with different mirror configurations, with their optical properties described by the scan mirror model. The scan mirrors can contain a contaminant layer. On the right, the optical bench module is depicted, which includes the telescope, the predisperser prism, and the science channel and PMD detectors. The crossed circle indicates the polarization direction of the PMD beam after internal reflection inside the prism (into the paper). The optical train between the predisperser prism and the PMD and science channel detectors has been omitted; for details, we refer to Gottwald and Bovensmann (2011).

Table 1. SCIAMACHY PMDs, their spectral range, average wavelength, reference wavelength, corresponding SCIAMACHY science channel and spectrally averaged OBM Mueller matrix elements for the PMDs.

\begin{tabular}{lrrrrrrrr}
\hline PMD & $\begin{array}{r}\text { Range } \\
(\mathrm{nm})\end{array}$ & $\begin{array}{r}\langle\lambda\rangle \\
(\mathrm{nm})\end{array}$ & $\lambda_{C}$ & Channel & $\left\langle M_{1}\right\rangle^{\mathrm{PD}}$ & $\left\langle\mu_{2}\right\rangle$ & $\left\langle\mu_{3}\right\rangle$ & $\left\langle\mu_{4}\right\rangle$ \\
\hline 1 & $305-385$ & 352 & 370 & 2 & 0.17 & -0.86 & -0.004 & -0.48 \\
2 & $430-550$ & 484 & 480 & 3 & 0.02 & -0.92 & 0.013 & -0.30 \\
3 & $590-720$ & 656 & 655 & 4 & 0.016 & -0.94 & 0.013 & -0.20 \\
4 & $780-920$ & 852 & 850 & 5 & 0.035 & -0.95 & 0.018 & -0.11 \\
5 & $1400-1750$ & 1570 & 1555 & 6 & 0.099 & -0.97 & 0.005 & 00.11 \\
7 & $780-920$ & 854 & 850 & 5 & 0.07 & 0.10 & 0.94 & 0.33 \\
\hline
\end{tabular}

tors, respectively. The $\mu_{n, i}^{\mathrm{P}, \mathrm{D}}$ are the end-to-end Mueller vector elements and vary with observation mode and scan angle. The factor $\mu_{1}^{\mathrm{PD}}$ is an additional in-flight calibration factor that accounts for calibration offsets in the relative PMD to science channel response to unpolarized light. Assuming that the polarization and the polarization sensitivity varies sufficiently slowly with wavelength, this equation can be further simplified:

$S^{\mathrm{P}}=\mu_{1}^{\mathrm{PD}} \cdot \sum_{i} S_{i}^{\mathrm{D}} M_{1, i}^{\mathrm{PD}} \frac{1+\left\langle\mu_{2}^{\mathrm{P}}\right\rangle q+\left\langle\mu_{3}^{\mathrm{P}}\right\rangle u}{1+\left\langle\mu_{2}^{\mathrm{D}}\right\rangle q+\left\langle\mu_{3}^{\mathrm{D}}\right\rangle u}$.

The quantities in the angular brackets are now wavelength independent and refer to the intensity-weighted spectral average of the polarization sensitivities:

$\left\langle\mu_{n}^{\mathrm{P}, \mathrm{D}}\right\rangle=\frac{1}{S^{\mathrm{D}}} \sum_{i} S_{i}^{\mathrm{D}} M_{1, i}^{\mathrm{PD}} \mu_{n i}^{\mathrm{P}, \mathrm{D}}, \quad n=2,3$ with

$S^{\mathrm{D}}=\sum_{i} S_{i}^{\mathrm{D}} M_{1, i}^{\mathrm{PD}}$

The term $S^{\mathrm{D}}$ is also called the "virtual sum" and describes the expected PMD signal for zero polarization, given the science channel signal and the relative detector responses. With Eq. (10) a polarization signal, $P$, can be defined as the ratio of the PMD signal to the virtual sum,

$P \equiv \mu_{1}^{\mathrm{PD}} \frac{S^{\mathrm{P}}}{S^{\mathrm{D}}} \approx \frac{1+\left\langle\mu_{2}^{\mathrm{P}}\right\rangle q+\left\langle\mu_{3}^{\mathrm{P}}\right\rangle u}{1+\left\langle\mu_{2}^{\mathrm{D}}\right\rangle q+\left\langle\mu_{3}^{\mathrm{D}}\right\rangle u}$,

which depends on polarization only.

Given known calibration constants and a relationship between $q$ and $u$, or a known value for $u$, Eqs. (6) or (11) can be solved and result in a value for $q$ which can be interpreted both as the signal-weighted average over the spectral width 
of the PMD and as its value at a representative wavelength ${ }^{1}$. This wavelength can be determined via

$$
\langle\lambda\rangle=\frac{1}{S^{\mathrm{D}}} \sum_{i} \lambda_{i} S_{i}\left(M_{1, i}^{\mathrm{PD}}\right)_{i} .
$$

On the other hand, if the polarization values at the representative wavelength are known, Eq. (11) enables the determination of the average $\left\langle\mu_{n}^{\mathrm{P}}\right\rangle$ for the PMDs and the unpolarized offset $\mu_{1}^{\mathrm{PD}}$. In the next section, the details of the calibration approach are laid out.

\section{Calibration model and data}

The calibration approach taken here exploits a phenomenological relationship between the measured reflectance and the depolarization. In general it should be possible to find a maximum polarization value for any unique observation geometry defined by the line-of-sight elevation angle $\left(\theta_{\mathrm{LOS}}\right)$, the SZA $\left(\theta_{0}\right)$ and the relative azimuth between the Sun and line of sight $(\phi)$. The maximum value is typically given by pure Rayleigh scattering. Scattering off aerosol or cloud droplets or the surface decrease the polarization in most cases, with the notable exceptions being, for instance, rainbow or glory effects generated by water or ice clouds, respectively. In clear sky conditions over the ocean, sunglint can add substantial polarization. This effect is largest close to the principal plane, when SZA and elevation angle are approximately equal and the relative azimuth is small. It depends on wind speed and direction, which influence the distribution, direction and form of waves. Two possible strategies emerge from these considerations: one would be to estimate the maximum polarization value for a given observation geometry and to devise a way to extrapolate the measurements to this point. This strategy resembles a modification and extension of the approach taken in Krijger et al. (2004) to validate polarization measurements with GOME. The other strategy would be to establish an adequate model that emulates the reflectance vs. polarization relationship in an averaged sense. These two strategies are explored in detail in the following.

\subsection{Data quality criteria}

Before the actual analysis, it is necessary to consider in detail the data selection criteria. Rainbow or glory effects and strong specular reflection destroy the assumed depolarization-reflectance relationships. Therefore, in nadir, the observation geometries where such effects are likely to occur are excluded. In limb, the data considered are typically above cloud layers, and specular reflection cannot be directly

\footnotetext{
${ }^{1}$ This is not quite true for the case of PMD 1, where the average wavelength of the PMD signal is around $350 \mathrm{~nm}$, but the polarization does not vary monotonically around this point. The wavelength matching the average polarization is closer to $\sim 370 \mathrm{~nm}$.
}

observed, such that these effects are considered to be negligible. However, in high-latitude winter it is possible that polar stratospheric clouds (PSCs) occur, which cause highly variable reflectance and polarization. A simple PSC detection algorithm, similar to the one described in von Savigny et al. (2005), was implemented in order to filter out data points with PSCs. In both measurement modes, data with high SZA larger than $80^{\circ}$ are rejected, and upper limits on $|\cos (\theta)|$ of 0.8 and 0.98 are imposed on limb and nadir data, respectively. Also, data where the subsatellite point is inside the South Atlantic Anomaly (SAA) region are excluded, because highly energetic particles are likely to affect the detectors or readout electronics severely and create "hot signals". In addition, quality criteria on the state of the instrument are imposed. For instance, data shortly after a decontamination period are not considered for this analysis.

\subsubsection{The limiting model}

The vector radiative transfer model SCIATRAN (Rozanov et al., 2014) can be used to calculate, for each individual data point, the limiting values for $q$ and $u$, i.e., the maximum possible polarization. For nadir over land and for limb, the limiting model is considered to be multiple Rayleigh scattering over a dark $($ albedo $=0)$ surface. Over ocean, in addition to Rayleigh scattering, an isotropic Cox-Munk parameterization (Cox and Munk, 1954) with a wind speed parameter set to $5 \mathrm{~ms}^{-1}$ is used to model the bidirectional reflectance distribution function (BRDF). The parameters set to define the water leaving reflectance and white cap contributions are the default parameters given in SCIATRAN version 3.5 (Rozanov and Rozanov, 2016). A correction for atmospheric refraction is employed for limb and nadir simulations. Nadir data were simulated with a pseudo-spherical geometry; i.e., the solar beam is modeled in a spherical shell atmosphere and the scattered beam in a plane-parallel one. In limb, a spherical atmosphere is assumed for both cases, although for technical reasons the multiply scattered part of the beam is approximated by a plane-parallel atmosphere. This leads to approximation errors in reflectance and polarization, because the contribution of multiple scattering is overestimated.

Absorption by trace gases is modeled using a climatology for trace gas concentrations according to McLinden et al. (2010) for continuum absorbers and Sinnhuber et al. (2009) for line absorbers. Settings for the temperature dependence of, for example, $\mathrm{O}_{3}$ cross sections are the default ones employed in SCIATRAN. Note that the exact modeling of atmospheric trace gas profiles is not too critical here, because in most cases the average PMD wavelengths are outside or only within weak $\left(\mathrm{O}_{3}\right.$ or $\left.\mathrm{H}_{2} \mathrm{O}\right)$ absorption bands. The Stokes vector was calculated for fixed wavelengths representing the average PMD wavelengths, integrating over a typical band width of 1 to $5 \mathrm{~nm}$. The RTM values have been generated in latitude bands of $10^{\circ}$ and for each month of the year and are mapped into a regular grid in $\left(\theta_{\mathrm{LOS}}, \theta_{0}, \phi\right)$. Eventually, by 
means of linear interpolation (in observation geometry only), a reference Stokes vector for the limiting RTM is available for each data point.

\subsubsection{The extrapolation method}

The data points for a given time period are now grouped according to the relevant scan angle (the position of the ESM mirror in nadir, the position of the ASM mirror for a fixed tangent height in limb) and in cells of ( $\left.q_{\mathrm{RTM}}, u_{\mathrm{RTM}}\right)$ with a typical width of 0.01 in each direction ( 0.02 for limb), as a function of the RTM normalized average reflectance $R / R_{\mathrm{RTM}}$ at the PMD central wavelength. The subscript RTM stands for the appropriate limiting model. The average reflectance is defined here as

$R=\pi \frac{1}{N} \sum_{i} \frac{I}{I_{0}} \quad\left|\lambda_{i}-\lambda_{C}\right| \leq \delta \lambda$,

where $I$ is the measured intensity and $I_{0}$ the solar irradiance at each spectral pixel $i$ within a window of width $\delta \lambda$ around the central wavelength $\lambda_{C}$ given in Table 1 . The width $\Delta \lambda$ is between 1 and $5 \mathrm{~nm}$, depending on wavelength, and $N$ is the number of valid spectral points inside this window. The ( $q_{\mathrm{RTM}}, u_{\mathrm{RTM}}$ ) cells are small enough that the viewing angles in each vary only slightly. In each cell, the variation in the polarization signal $P$ with $\delta R / R_{\mathrm{RTM}}=R / R_{\mathrm{RTM}}-1$ can be fitted with a function of the following form:

$f\left(\frac{\delta R}{R_{\mathrm{RTM}}}\right)=p_{0}+\frac{p_{1}}{1+p_{2} \frac{\delta R}{R_{\mathrm{RTM}}}}$,

with three fit parameters $p_{i}$. This functional form is phenomenological but motivated by the expected behavior of depolarization induced by a single physical process, for instance the increase in surface albedo or of the aerosol concentration of a fixed aerosol type. The depolarization of each individual process would then be governed by the parameter $p_{2}$. In reality, multiple processes would contribute and cause considerable scatter. The underlying assumption here is that this scatter should decrease as the measured reflectance approaches the RTM limit since each possible process can only have a small effect, such that the data should converge to a well-defined slope. The polarization signal corresponding to the maximum (RTM) polarization in the center of the cell is then

$P(0)=f\left(R=R_{\mathrm{RTM}}\right) \equiv P\left(q_{\mathrm{RTM}}, u_{\mathrm{RTM}}\right)=p_{0}+p_{1}$.

For this fit, the reflectance is corrected for polarization with the in-flight MMEs obtained from the scan mirror model and the on-ground OBM Mueller vector for the corresponding wavelengths. Since in this step of the analysis the actual polarization values for each data point data are not yet available, the polarization values used in the correction are the RTM values themselves; i.e., each data point is corrected for

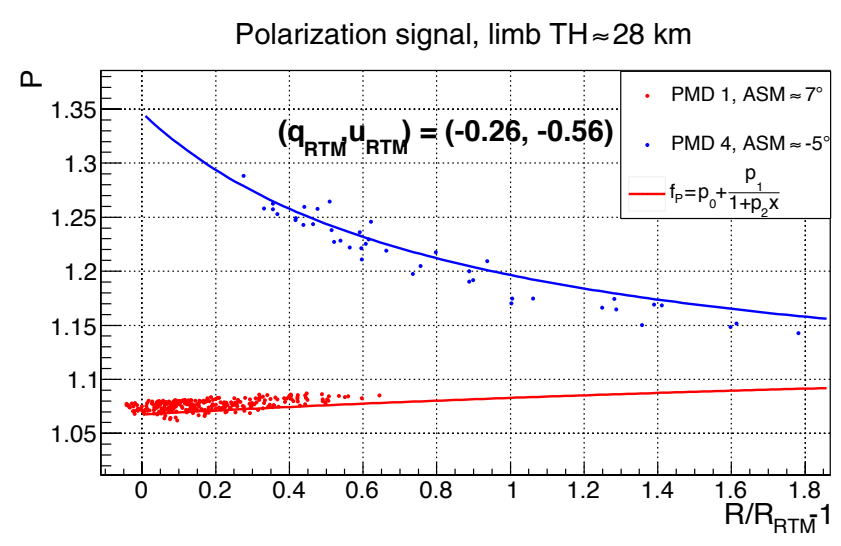

Figure 3. Examples for the extrapolation method for limb, for a fixed cell of $\left(q_{\mathrm{RTM}}, u_{\mathrm{RTM}}\right)$. Blue points are for PMD 4 , and red points are for PMD 1; the corresponding curves show the fitted functions.

the maximum polarization $\left(q_{\mathrm{RTM}}, u_{\mathrm{RTM}}\right)$ at $R=R_{\mathrm{RTM}}$ :

$c_{\mathrm{RTM}}^{P}=\frac{1}{1+\mu_{2} q_{\mathrm{RTM}}+\mu_{3} u_{\mathrm{RTM}}}$.

This means that the reflectance curve will be somewhat distorted towards the high reflectance tails. It has been verified, though, that this has no influence on the estimate of the limit towards the low reflectance values, where the correction should be closer to the truth.

In Fig. 3 the method is demonstrated for the case of PMD 1 and PMD 4 in limb.

Note that for the examples in the figure the viewing geometries for PMD 1 and 4 are not the same, despite the RTM polarization values being the same. Also, in order to get a sufficient amount and leverage of data for the fit, the data have to be collected over the period of at least 1 month. To avoid large extrapolation errors it is also vital to disregard cells where the minimum reflectance is too far away from the RTM limit, which happens very often at higher wavelengths in nadir over land due to the contribution of surface reflectance.

The fitted maximum polarization values fill a certain range in the $(q, u)$ plane which is determined by scan angle, scattering geometries and the period of time integrated over. Here, this period is normally 1 calendar year, except for 2002, when it starts only in August after the end of the commissioning phase, and for 2012, when the mission ended in April. A typical example for the maximum polarization signal obtained in nadir, 2004, is given in Fig. 4 for PMDs 1 and 4.

\subsubsection{The lookup table method}

The second approach is only realized for limb, where a large simulation data set is available for a subset of the limb observation geometries, comprising a range of aerosol profiles with different types, different cloud layers and types, and val- 

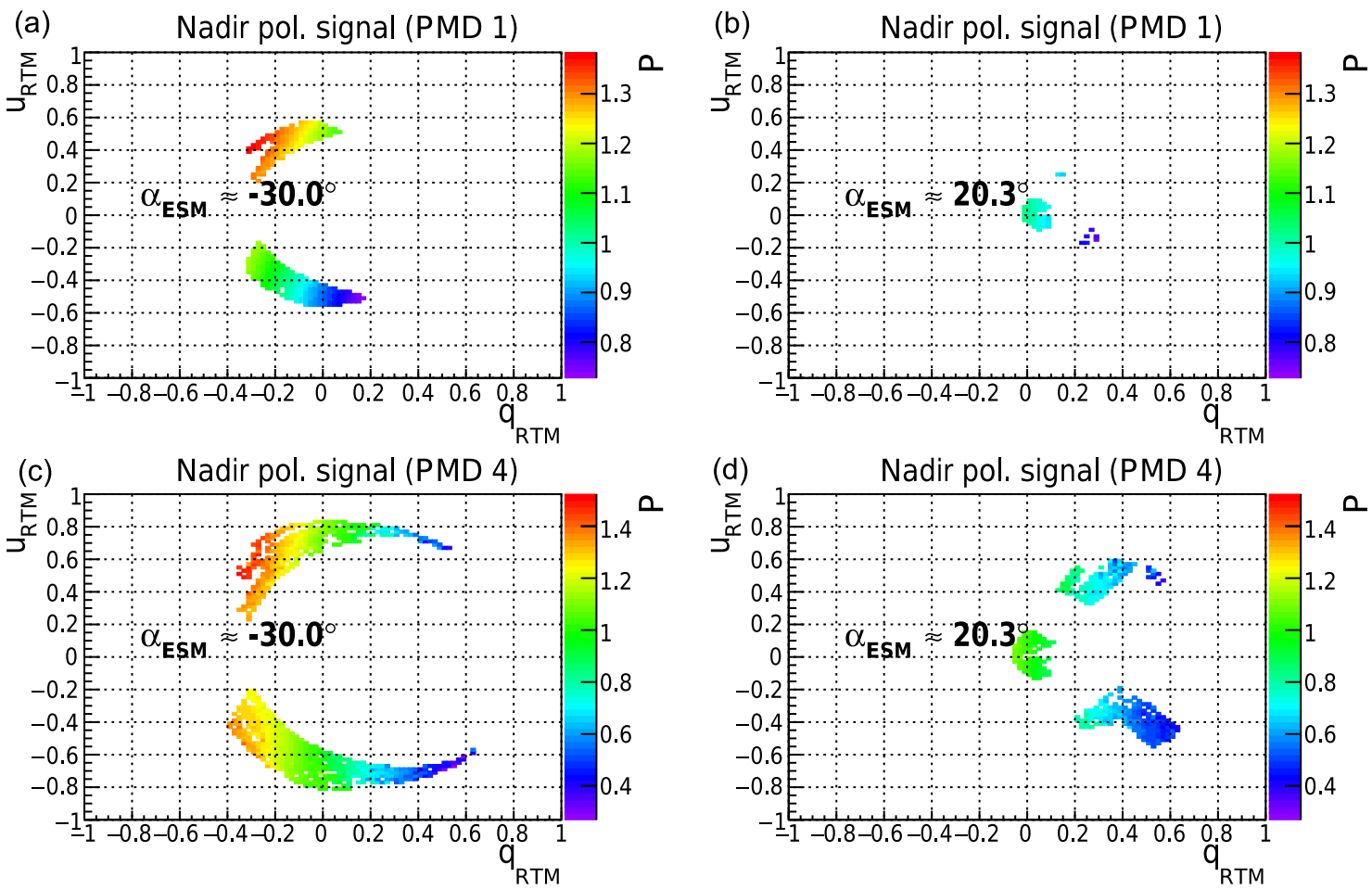

Figure 4. Polarization signals for nadir (rainbow color code) vs. $q_{\mathrm{RTM}}\left(x\right.$ axis) and $u_{\mathrm{RTM}}(y$ axis) over ocean in 2004 from the extrapolation method for PMD 1 (a, b), PMD 4 (c, d) and for two different ESM mirror positions during the nadir scan (a, c: east; b, d: west).

ues for the Lambertian albedo between 0 and 0.8 . The details on this simulation data set are already described in Liebing et al. (2013). In order to make these simulations applicable to all limb observation geometries, the simulated data are once again grouped according to observation geometry and averaged over a smaller subset of all scenarios, including all but the highest values of stratospheric aerosol optical depth, which are unrealistic for the time period considered here. An arithmetic average of $q / q_{\mathrm{RTM}}\left(u / u_{\mathrm{RTM}}\right)$ of all scenarios is determined as a function of $R / R_{\mathrm{RTM}}$, where the subscript RTM refers to the limiting RTM value for pure Rayleigh scattering. After smoothing, this average is mapped as a function of $q_{\mathrm{RTM}}\left(u_{\mathrm{RTM}}\right)$, such that for each PMD a two-dimensional lookup table (LUT) is available that delivers the depolarization in each Stokes component as a function of its maximum RTM value. The approach is illustrated in Fig. 5 for PMDs 1 and 4.

On the left side, the individual simulation points and their averaged values for a fixed TH $(28 \mathrm{~km})$ and fixed viewing geometry are shown; the right side shows the two-dimensional LUT built from a range of viewing geometries. From this LUT, a value for $\left(q_{\mathrm{LUT}}, u_{\mathrm{LUT}}\right)$ can be determined for each data point given in terms of ( $\left.q_{\mathrm{RTM}}, u_{\mathrm{RTM}}, R / R_{\mathrm{RTM}}\right)$ :

$p_{\mathrm{LUT}}=p_{\mathrm{RTM}}\left\langle\frac{p}{p_{\mathrm{RTM}}}\right\rangle\left(p_{\mathrm{RTM}}, R / R_{\mathrm{RTM}}\right) p=q, u$.
Also for this method, the reflectance values are corrected for polarization using Eq. (16), except that here the RTM polarization values are replaced by the LUT polarization values, and an iteration step is performed to arrive at the final value for $p_{\mathrm{LUT}}$, given the polarization-corrected reflectance.

These data are again collected in cells of $\left(q_{\mathrm{LUT}}, u_{\mathrm{LUT}}\right)$ and in bins of ASM mirror angle and tangent height and averaged over 1 year. Examples for the resulting distributions are given on the right side of Fig. 6, together with the corresponding results from the extrapolation method on the left, once again for PMDs 1 and 4.

Comparing the extrapolation- and LUT-method values, it is evident that the sampling is much denser for the LUT method but that the range of $(q, u)$ values covered is larger with the extrapolation method - naturally because the extrapolation method by definition always selects the maximum possible polarization values. The LUT method is highly dependent on the assumptions used in the simulation of the different scenarios, e.g., aerosol properties and profiles, and on the scenario selections and averaging method applied to prepare the LUTs. Resulting calibration values may therefore be considerably model dependent. The extrapolation method on the other hand is only marginally model dependent since the basic mechanisms of Rayleigh and ocean surface scattering are relatively well known and can be accurately computed. The potential pitfall lies in the assumption on how representative the limiting polarization condition is and how well it 
(a)

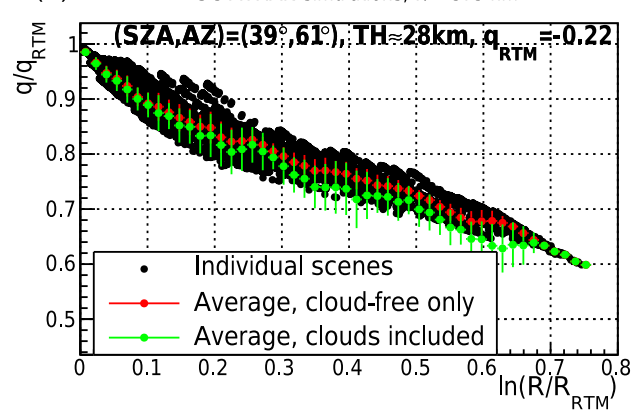

(c)

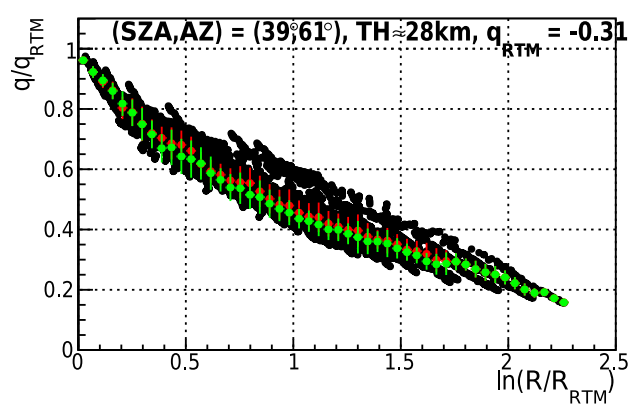

(b)

$\mathrm{q}, \lambda=375 \mathrm{~nm}$, orbit phase $<0.7, \mathrm{TH} \# 8$

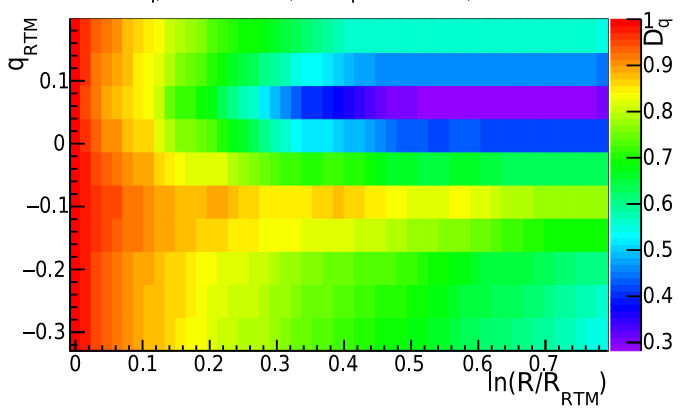

(d) $\quad$, $\lambda=850 \mathrm{~nm}$, orbit phase $<0.7, \mathrm{TH} \# 8$

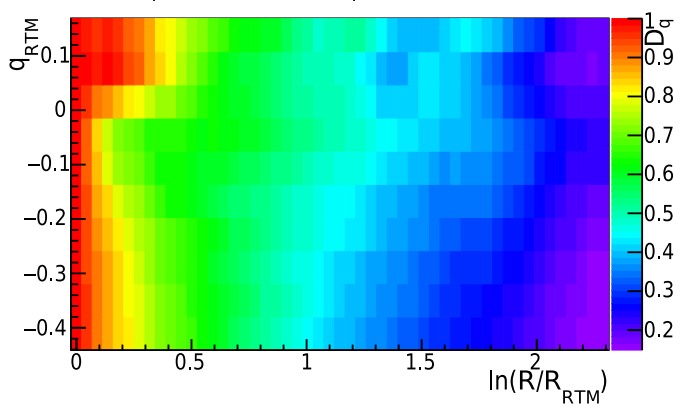

Figure 5. Example for the derivation of LUTs for the depolarization in limb geometry, TH $\approx 28 \mathrm{~km}$. (a) and (b) are for $375 \mathrm{~nm}$ (i.e., PMD 1); (c) and (d) are for $850 \mathrm{~nm}$ (i.e, PMDs 4 and 7). On the left the depolarization for a fixed geometry is plotted vs. $\log \left(R / R_{\mathrm{RTM}}\right)$; on the right the $2 \mathrm{D}$ LUTs are shown as $q / q_{\mathrm{RTM}}=f\left(q_{\mathrm{RTM}}, \log \left(R / R_{\mathrm{RTM}}\right)\right)$ in a rainbow color scale.

(a)

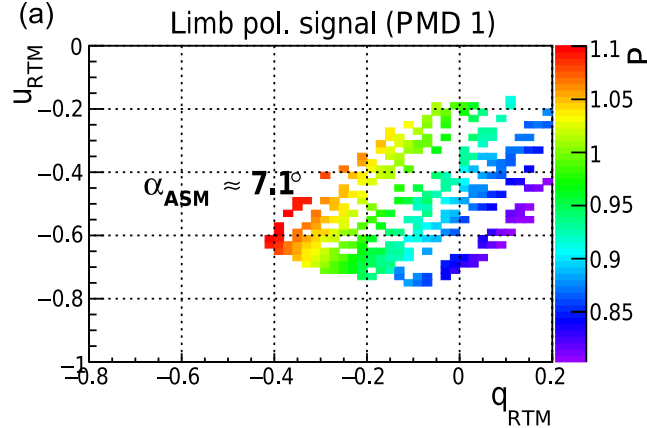

(c)

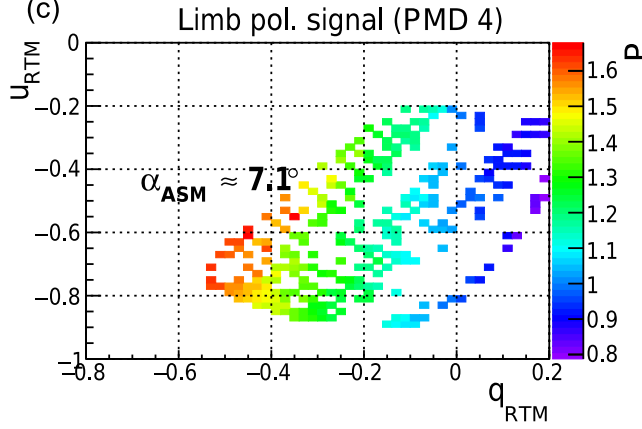

(b)

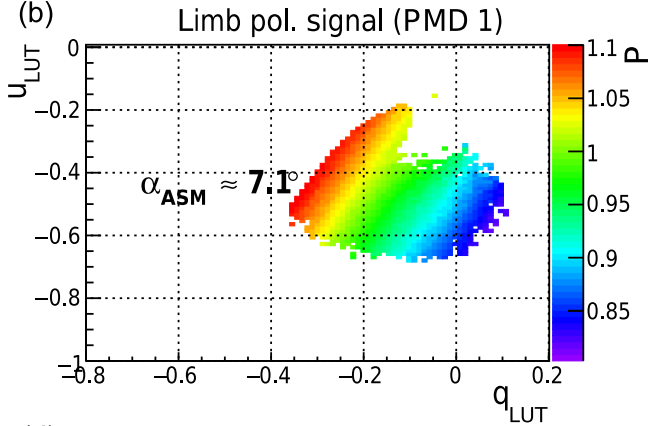

(d)

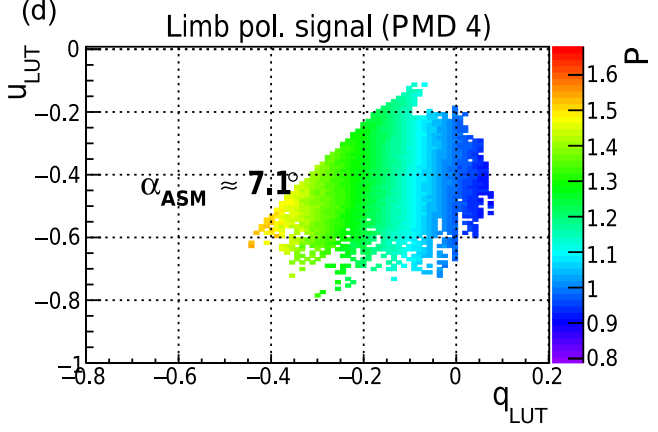

Figure 6. Polarization signals for limb (rainbow color code) in 2004 vs. $q_{\mathrm{RTM}(\mathrm{LUT})}\left(x\right.$ axis) and $u_{\mathrm{RTM}(\mathrm{LUT})}(y$ axis), TH $\approx 28 \mathrm{~km}$, from the extrapolation method $(\mathbf{a}, \mathbf{c})$ and the LUT method $(\mathbf{b}, \mathbf{d})$ for PMD $1(\mathbf{a}, \mathbf{b})$, PMD $4(\mathbf{c}, \mathbf{d})$ and for an ASM mirror angle of about $+7^{\circ}$. 
Channel 2 end-to-end MMEs

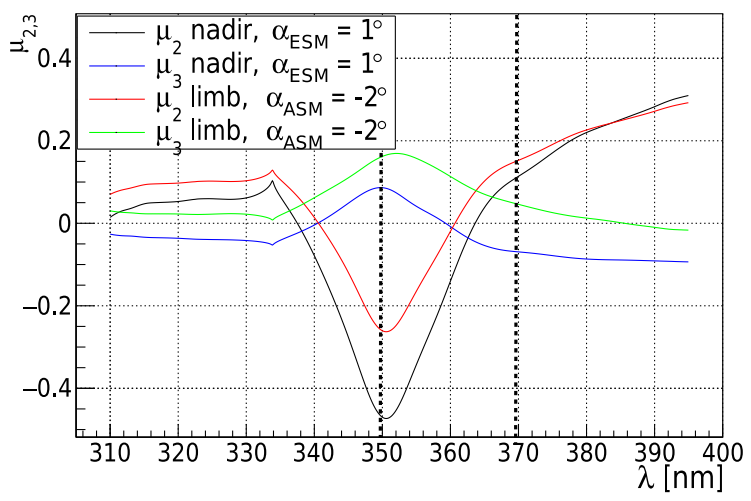

Figure 7. Polarized end-to-end MMEs $\left(\mu_{2,3}\right)$ for nadir (black: $\mu_{2}$; blue: $\mu_{3}$ ) and limb (red: $\mu_{2}$; green: $\mu_{3}$ ) calculated with the scan mirror model for August 2002. The MMEs are given in the atmospheric Stokes frame. The thick dashed vertical lines indicate the two spectral points used to build the polarization signal (see text).

can be reproduced by the fit with Eq. (14). For each individual case the resulting errors can hardly be estimated since many different effects may cancel or enhance each other, but the comparison of the calibration parameters obtained by the two methods can provide a viable estimate of the typical uncertainties involved in this analysis.

\subsection{The polarization feature at $350 \mathrm{~nm}$}

With the polarization signal as defined in Eq. (11), i.e., the ratio of PMD to science channel signals, it is only possible to derive the in-flight MMEs for the PMDs. There is no independent information on the science channel MMEs. A particularly strong polarization feature in SCIAMACHY's channel 2, located at around $350 \mathrm{~nm}$, opens up the possibility of deriving information about the in-flight polarization sensitivity at this wavelength and as such enables a direct comparison of science channel and PMD 1 behavior at almost the same wavelength. Figure 7 shows the polarized end-to-end MMEs (nadir and limb) for channel 2 as a function of wavelength. The properties of this feature indicate the presence of a socalled Wood's anomaly (see, e.g., Maystre, 2012) of the grating.

The feature at $350 \mathrm{~nm}$ was recognized as a potential noncompliance with respect to the instrument specification. However, the high throughput of this grating resulted in its selection nevertheless. Its sensitivity to polarization provides independent information on the polarization response in channel 2 but may contribute to noise or bias in trace gas retrievals if not perfectly accounted for.

The polarization-sensitive signal can be constructed by comparing two relatively close spectral points with very different polarization sensitivity. An obvious choice would be a point around $350 \mathrm{~nm}$ close to the minimum of $\mu_{2}$ and another one outside the feature at $370 \mathrm{~nm}$ where both MMEs are relatively small. Points to the left of the feature are too sensitive to $\mathrm{O}_{3}$ concentrations and therefore not considered. The polarization signal is

$$
\begin{gathered}
P_{350}\left(\frac{R(370 \mathrm{~nm})}{R_{\mathrm{RTM}}(370 \mathrm{~nm})}\right) \equiv \frac{R(350 \mathrm{~nm}) / R(370 \mathrm{~nm})}{R_{\mathrm{RTM}}(350 \mathrm{~nm}) / R_{\mathrm{RTM}}(370 \mathrm{~nm})} \\
=C\left(\frac{R(370 \mathrm{~nm})}{R_{\mathrm{RTM}}(370 \mathrm{~nm})}\right) \frac{1+\mu_{2}^{350} q(350 \mathrm{~nm})+\mu_{3}^{350} u(350 \mathrm{~nm})}{1+\mu_{2}^{370} q(370 \mathrm{~nm})+\mu_{3}^{370} u(370 \mathrm{~nm})} .
\end{gathered}
$$

The factor $C$ accounts for a possible calibration offset as well as a change in spectral slope between 350 and $370 \mathrm{~nm}$ with atmospheric or surface conditions. This is in contrast to the PMD to science channel polarization signal, which by definition depends only on the polarization. For $P_{350}$, this is only the case for the point where $R(370 \mathrm{~nm})=R_{\mathrm{RTM}}(370 \mathrm{~nm})$, i.e., at the RTM limit. This also implies that the LUT method cannot be applied for this polarization signal.

\subsection{Derivation of in-flight Mueller matrix elements and their errors}

\subsubsection{Fit of end-to-end MMEs}

The result of the previous analysis step are three-dimensional distributions of the polarization signal in $(q, u, \alpha)$, which can be modeled as (see also Eq. 11)

$P(u, q, \alpha)=\left\langle\mu_{1}\right\rangle(\alpha) \frac{1+\left\langle\mu_{2}^{P}\right\rangle(\alpha) q+\left\langle\mu_{3}^{P}\right\rangle(\alpha) u}{1+\left\langle\mu_{2}^{D}\right\rangle(\alpha) q+\left\langle\mu_{3}^{D}\right\rangle(\alpha) u}$,

where $\alpha$ is the scan angle and

$\left\langle\mu_{i}\right\rangle(\alpha)=p_{i 0}+p_{i 1} \alpha+p_{i 2} \alpha^{2}, \quad p_{i 2} \equiv 0$ for limb.

Due to the limited range of $(q, u)$ values covered, it is not possible to simultaneously fit all five MMEs. The aim is instead to fit the PMD parameters and the unpolarized scale factor $\left\langle\mu_{1}\right\rangle$ in a linear fit. This is achieved by correcting the polarization signal for the science channel contribution by simply multiplying each cell value by a factor:

$C^{D}=1+\left\langle\mu_{2}^{D}\right\rangle(\alpha) q_{\mathrm{RTM}, \mathrm{LUT}}+\left\langle\mu_{3}^{D}\right\rangle(\alpha) u_{\mathrm{RTM}, \mathrm{LUT}}$.

For the channel 2 feature $\mu_{i}^{350}$ corresponds to $\mu_{i}^{P}$, and the $\mu_{i}^{D}$ 's are to be replaced by the MMEs at $370 \mathrm{~nm}, \mu_{i}^{370}$. The RTM polarization values at $370 \mathrm{~nm}$ have to be used for the correction of the reflectance.

The fit is performed as a weighted least squares fit. The fit parameters are the $p_{i j}$ in Eq. (20). The statistical errors of the fit parameters and their correlations are determined by the extrapolation fit error (extrapolation method) or by the error of the mean (LUT method) in each cell and the density and leverage of the 2D $(q, u)$ distribution. Figure 4 can already give an impression about the uneven distribution of data cells for different scan angles in nadir, and in Fig. 6 it should be pointed out that the limb data cover mostly only one quadrant 
of the possible $(q, u)$ range. Statistical uncertainties and correlations are therefore expected to increase with scan angle in nadir and to be larger in limb than in nadir. However, the statistical uncertainties are negligible compared to the systematic ones. This is indicated already by the typical reduced $\chi^{2}$ of the fit, which is significantly above 2 for all but PMD 1 and the channel 2 feature.

\subsubsection{Systematic errors}

In order to obtain a realistic estimate of the systematic uncertainties related to this analysis, it is possible to vary some of its critical assumptions and compare the results. The normalization uncertainty in the calibration of the reflectance would alter the $x$ axis of the polarization-reflectance curve and shift the RTM limit point in all observation modes and in both methods. There is no official estimate yet available for the calibration uncertainty with the scan mirror model. Comparisons with previous level 1 data versions and of nadir data with MERIS data, the fitted values for the calibration factor $\mu_{1}$ and the possible stray light contamination in limb suggest that a value of about $\delta R / R \approx \pm 5 \%$ should cover the expected order of magnitude quite well. The systematic uncertainty due to the calibration error is estimated by shifting the reflectance of each data point by $\delta R$ and then repeating the entire chain of fits.

For limb data, an estimate of a theoretical model uncertainty arising from the plane-parallel approximation of the multiple scattering contribution on both reflectance and polarization is included in the total systematic error. The uncertainty has been estimated from a comparison between SCIATRAN and the MYSTIC (Emde and Mayer, 2007) Monte Carlo model (Rozanov et al., 2014). Its contribution to the systematic error of the polarization sensitivities is in general smaller than the error arising from the normalization uncertainty and is therefore not depicted explicitly.

In nadir over ocean, an obvious error source is the assumption on the wind speed for the ocean BRDF model. This wind speed is not meant to be representative but is rather meant to be the lower boundary against which the data are measured. The choice of $5 \mathrm{~m} \mathrm{~s}^{-1}$ was optimized by comparing RTM normalized reflectance distributions for different wind speeds. However, slightly lower or higher wind speeds also seemed viable. A wind-speed-related uncertainty for nadir can therefore be estimated from replacing the RTM reference values with those for wind speeds of 3 and $7 \mathrm{~m} \mathrm{~s}^{-1}$ and then again repeating the entire analysis chain and comparing the results.

For limb data it is not directly possible to vary the model assumptions because of the prohibitively large effort to generate a comprehensive RTM database. It is conceivable, though, that investigating the results obtained for a range of tangent heights could deliver a proxy for the model uncertainty. This is because certain model parameters maybe more appropriate at one TH than at another or because contribu- tions of albedo, stray light, etc. change with TH. The limb data are therefore analyzed for a range of THs between 25 and $35 \mathrm{~km}$ and compared to the reference value at $28 \mathrm{~km}$.

For each of these systematic error classes (reflectance normalization error, BRDF model error in nadir and TH variation in limb) a systematic uncertainty with respect to the reference model and data is derived by computing the standard deviation and the covariance as a function of scan angle for the end-to-end MMEs. The covariance between the derived $\mu_{2}$ and $\mu_{3}$ is also computed. The total covariance matrix is obtained as the squared sum of the statistical and each considered class of systematic error matrices. The combined covariance matrix is dominated by the systematic contribution, with strong (anti-)correlation prevailing between different scan angles for one MME or between $\mu_{2}$ and $\mu_{3}$ at a given scan angle. Those correlations are close to $\pm 100 \%$ in many cases and the correlation matrix turns out to be numerically degenerate when used in subsequent fits of the in-flight MMEs with the retarder model (see below). The covariance matrix is therefore "regularized" by scaling the off-diagonal elements by a factor 0.8 , thus allowing additional, unknown error components to destroy the almost perfect correlation between fit parameters.

Generally, studies on the sensitivity of both methods with respect to model input parameters and data selection criteria indicate that at wavelengths above $500 \mathrm{~nm}$ the results become rather unstable. At lower wavelengths the measurements are less influenced by the unknown atmospheric and surface conditions, such that results are more reliable. In the following, the discussion is therefore restricted to the results obtained from PMDs 1 and 2 and channel 2.

\subsubsection{Nadir results}

The fit results for the nadir end-to-end MMEs for the PMDs 1 and 2 and the channel 2 feature are displayed in Fig. 8.

The curves with the error bars depict the final results to be used for further analysis; these are the data over ocean. The different color error bars indicate the individual contribution from the reflectance normalization (magenta) and wind speed (green) variations to the total error. In the UV, the dominating source of error is the calibration uncertainty, while the wind speed uncertainty takes over at higher wavelengths. The results shown here were fitted to the nadir data over ocean only. The results for the data over land differ systematically from the data over ocean in most of the parameters, with increasing differences at longer wavelengths. In general, the data over land have to be regarded as unreliable due to their intrinsic sparsity - because of the above mentioned extrapolation errors as well as a sampling bias in $u$. The sampling is problematic because positive $u$ values prevail in northern latitudes and negative values in the Southern Hemisphere, where in general less landmass exists. This is why for this analysis here the data over land are disregarded. 
(a)

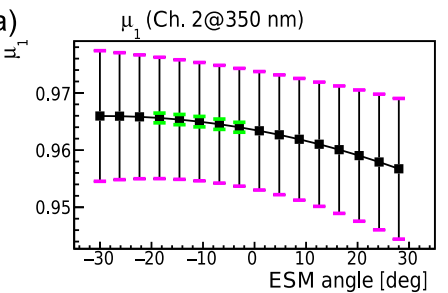

(b)

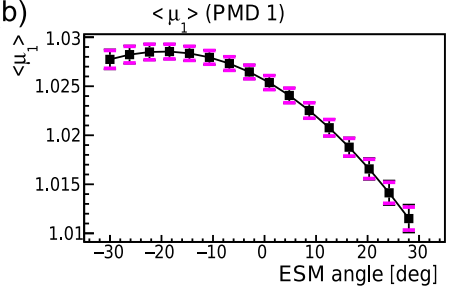

(c)

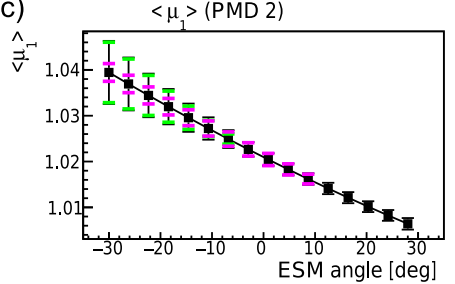

(d)

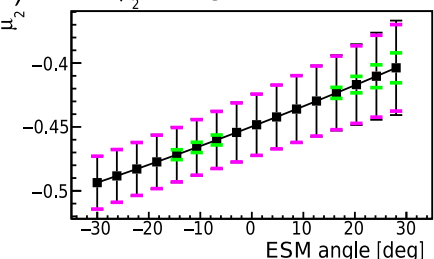

(e)

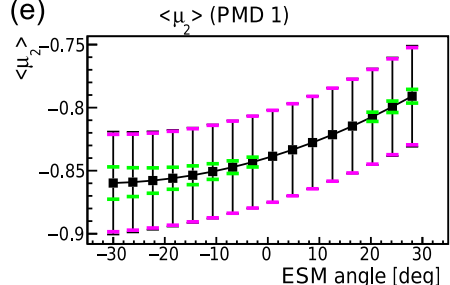

(f)

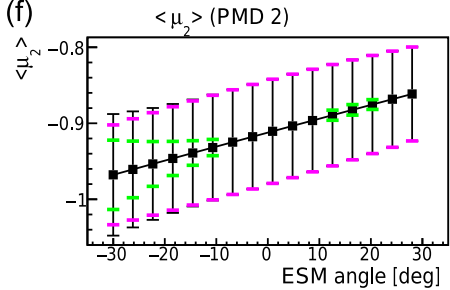

(g)

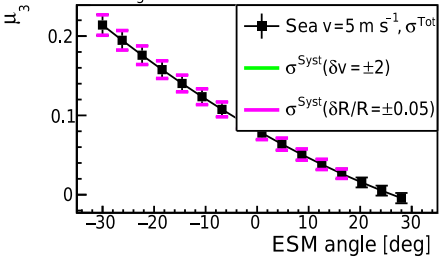

(h)

$<\mu_{3}>$ (PMD 1)
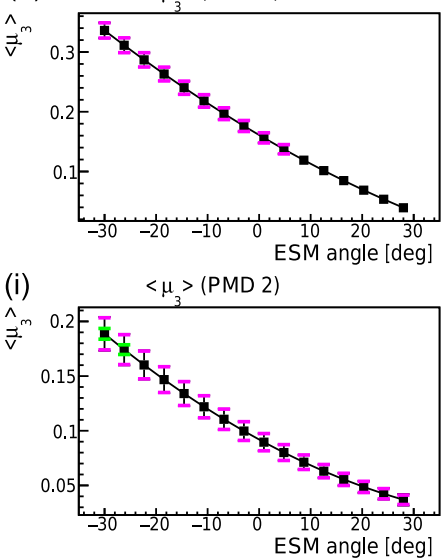

Figure 8. Final results for end-to-end MMEs vs. ESM angle for nadir over ocean in 2004 from the extrapolation method. Rows: (a, d, g) channel 2 feature, (b, e, h) PMD 1, (c, f, i) PMD 2; columns: (a, b, c) $\left\langle\mu_{1}\right\rangle,(\mathbf{d}, \mathbf{e}, \mathbf{f})\left\langle\mu_{2}^{P}\right\rangle$ and $(\mathbf{g}, \mathbf{h}, \mathbf{i})\left\langle\mu_{3}^{P}\right\rangle$. Black data points are the results with total errors; magenta and green error bars indicate the contribution from each class of systematic errors.

(a)

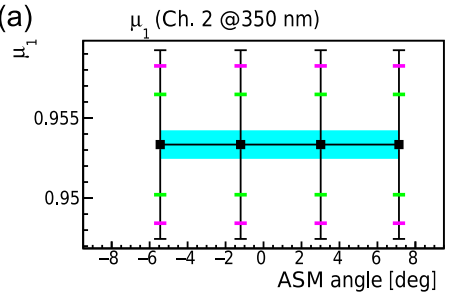

(b)

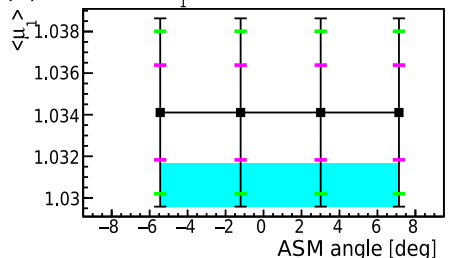

(c)

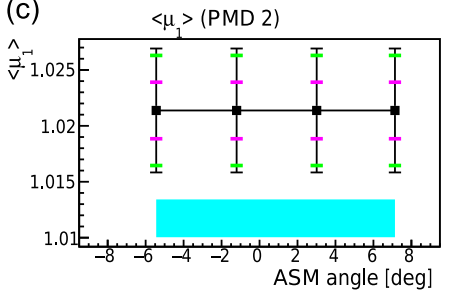

(d)

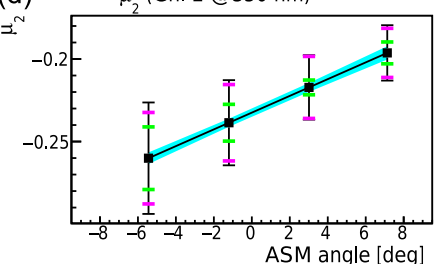

(e)

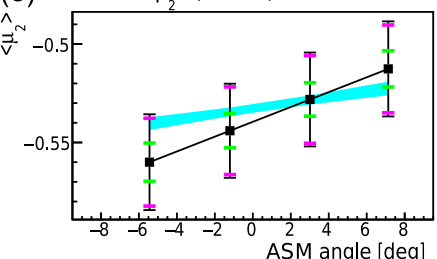

(f)

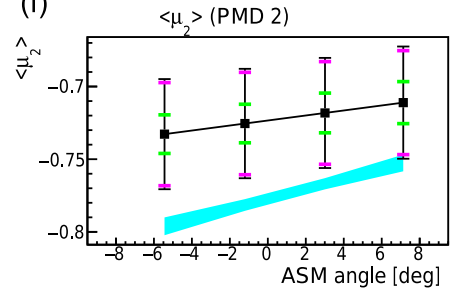

(g)

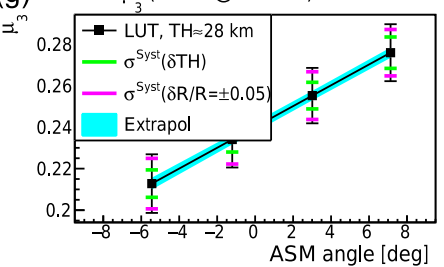

(h)

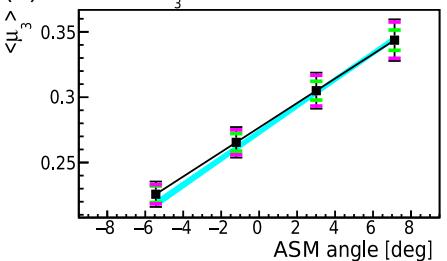

(i)

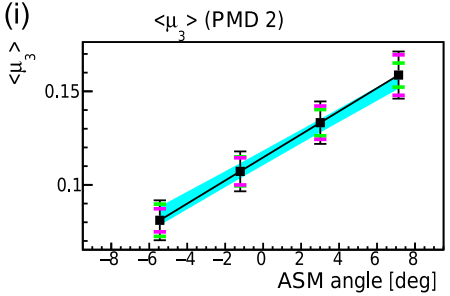

Figure 9. Final results vs. ASM angle for limb at a TH of $28 \mathrm{~km}$ in 2004 from the LUT method (PMDs) and extrapolation method (channel 2). Rows: (a, d, g) channel 2 feature, (b, e, h) PMD 1, (c, f, i) PMD 2; columns: (a, b, c) $\left\langle\mu_{1}\right\rangle$, (d, e, f) $\left\langle\mu_{2}^{P}\right\rangle$ and (g, h, i) $\left\langle\mu_{3}^{P}\right\rangle$. Black data points are the results with total errors; magenta and green error bars indicate the contribution from each class of systematic errors. The light-blue band shows the result from the extrapolation method with statistical errors only. 


\subsubsection{Limb results}

The fit results for the limb end-to-end MMEs for the PMDs 1 and 2 and the channel 2 feature are displayed in Fig. 9.

Here, the green error bars indicate the contribution of $\mathrm{TH}$ variation; the magenta ones are again the contribution of the normalization uncertainty. For the UV-VIS regions considered here, both contributions are similar in magnitude. The light-blue band is the result from the extrapolation method (statistical errors only). For PMD 1 and channel 2, the results are well within the systematic errors of the LUT method, while for PMD 2 the difference is slightly larger, at least for $\mu_{2}$. In general the differences between the two methods increase with wavelength, as expected because intrinsic errors of both methods increase.

For the fits to the limb data, the unpolarized calibration factor $\mu_{1}$ is assumed to be independent of scan angle. The unpolarized calibration parameter constitutes an important in-flight correction for the polarization determination but is treated as a nuisance parameter in this analysis. It may be scan-angle-dependent at short wavelengths, where the degradation parameters of the mirror model may introduce errors, but at higher wavelengths it is more likely to be constant. For the relatively small ASM scan angle range relevant for the limb data, leaving $\mu_{1}=$ constant warrants better stability of the fit parameters, especially at the higher wavelengths. For the wavelengths relevant for Fig. 9, letting $\mu_{1}(\alpha) \neq$ constant has only a small effect within the systematic errors.

\subsubsection{Comparison to the scan mirror model}

For both observation modes it was observed that the results become increasingly unreliable with increasing wavelength. For the following comparisons and analysis we therefore concentrate only on PMD $1(\sim 350 \mathrm{~nm})$, PMD $2(\sim 480 \mathrm{~nm})$ and the feature in channel 2 at $350 \mathrm{~nm}$. With the mirror model, the thickness and refractive index of the contaminant layers on the ESM and ASM mirrors can be determined from (solar) in-flight calibration data. Figure 10 shows the parameters of the current version of the mirror model, which have also been used for the most recent reprocessing of the level 1 data (version 9).

The ESM contaminant thickness increases steadily until about 2011 and then experiences a steep drop. The ASM contaminant thickness is rather small and almost constant, increases abruptly after a decontamination procedure in January 2009 and also drops steeply after 2011. The refractive index is assumed to be constant in time and the same for all mirrors. Its imaginary part is largest in the UV. Due to this enhanced absorption of UV light by the contaminant layer, the effect of mirror degradation is expected to be largest in the UV region.

The scan mirror model can be used to compute the end-toend MMEs for each observation mode, scan angle and point in time. The end-to-end Mueller vector according to Eq. (5) is
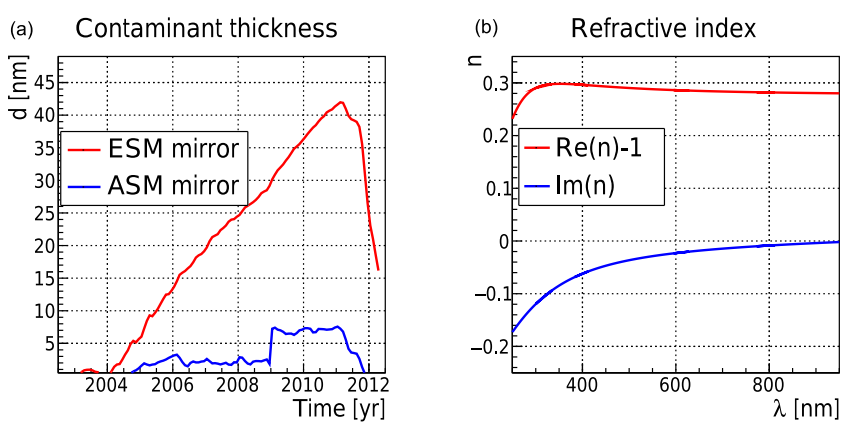

Figure 10. (a) Time dependence of contaminant layer thicknesses as determined from in-flight calibration data; red: ESM mirror; blue: ASM mirror. (b) The real (red, $\operatorname{Re}(n)-1$ is plotted) and imaginary (blue) part of the refractive index of the contaminant vs. wavelength.

the product of the OBM vector with the scan mirror Mueller matrix:

$\boldsymbol{\mu}_{\mathrm{N}, \mathrm{L}}(\alpha)=\boldsymbol{\mu}^{\mathrm{OBM}} \mathbf{M}(\alpha)$,

where the subscript $\mathrm{N}$ and $\mathrm{L}$ stand for nadir and limb, respectively. For the comparison, the mirror model values for the spectrally resolved PMD MMEs are averaged over wavelength using representative spectra for each month. As the wavelength dependence of the PMD MMEs is very slow or even constant, the averaging does not have a large impact.

Figure 11 shows how the scan mirror model compares to the nadir MMEs and Fig. 12 to the limb MMEs for the duration of the mission.

For nadir, the mirror model predicts rather constant values of $\mu_{2}$ over time and a significant increase in $\mu_{3}$. The values for $\mu_{2}$ reproduce the data quite well, although there seems to be a small but systematic decrease in the data during the first two-thirds of the mission. Note that due to the systematic nature of the in-flight errors, the interannual variation may be highly correlated, such that trends may be significant despite the large individual errors. The larger scan angle dependence in the data on the other hand may not be significant. The data follow the predicted trend in $\mu_{3}$ approximately, but in the UV there is also a distinct offset, even close to the start of the mission. The differences decrease with wavelength.

In limb the situation is very different. The mirror model predicts a significantly smaller $\mu_{3}$ and the magnitude of $\mu_{2}$ seems to be overestimated. The time dependence of the data is not reproduced, as differences grow with time. Comparing to the right panel of Fig. 10 it is tempting to suspect a correlation of the observed trends in the differences between data and model with those of the contaminant thicknesses. However, a direct relationship is not straight forward to discern. The large offset at the start of the mission, in particular in limb, cannot be related to possible errors in the degradation parameters, as the contamination at this point in time is prac- 

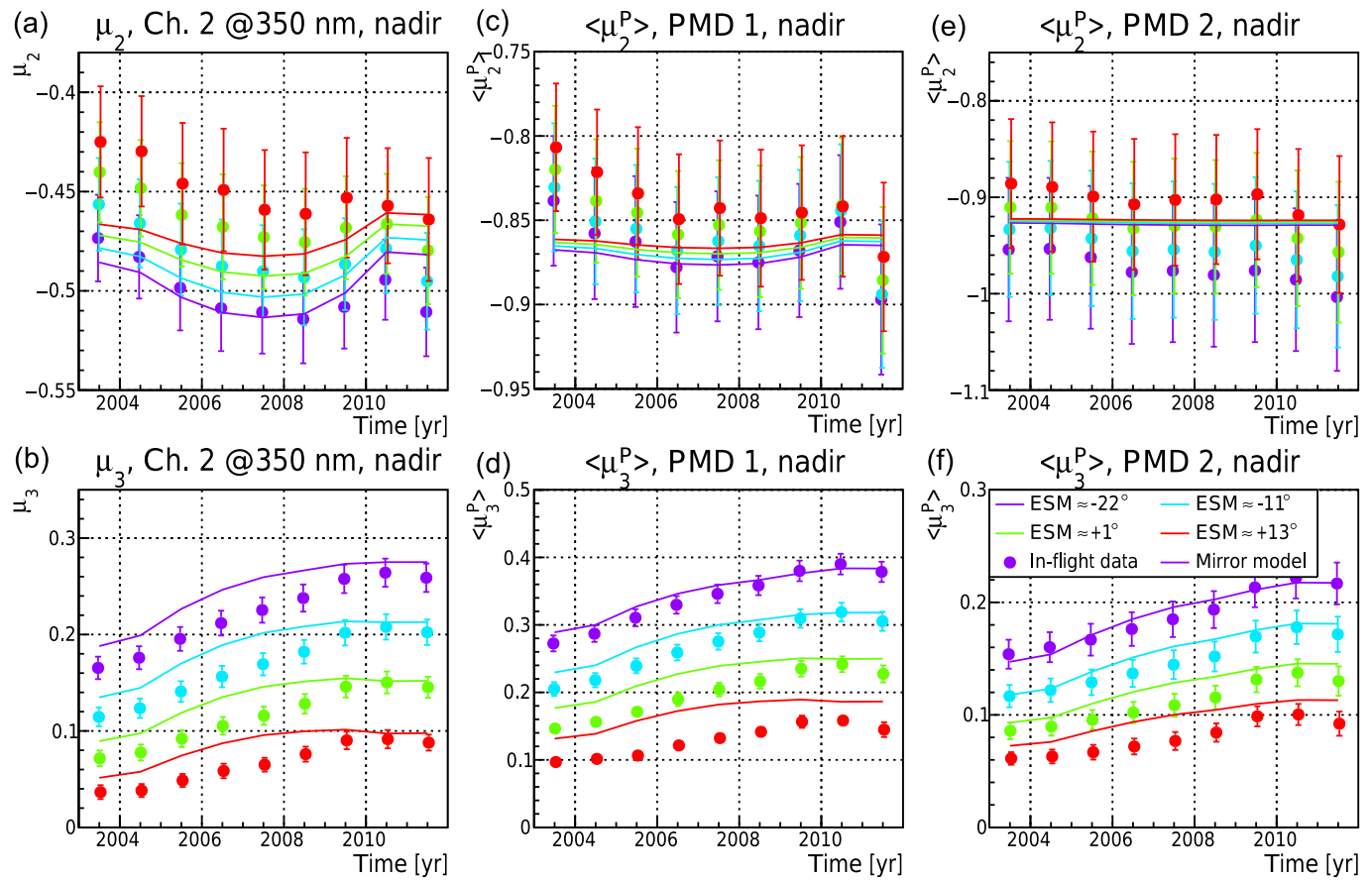

Figure 11. Time dependence of in-flight end-to-end Mueller matrix elements $\mu_{2}(\mathbf{a}, \mathbf{c}, \mathbf{e})$ and $\mu_{3}$ (b, d, f) for nadir for four selected ESM angles from measurements (data points) and the scan mirror model (lines). The error bars include statistical and total systematic errors. (a, b) Channel 2 feature; (c, d) PMD 1; (e, f) PMD 2. The color code signifies the ESM scan angle bin $\left(-22,-11,+1,+13^{\circ}\right)$, increasing on a rainbow color scale.
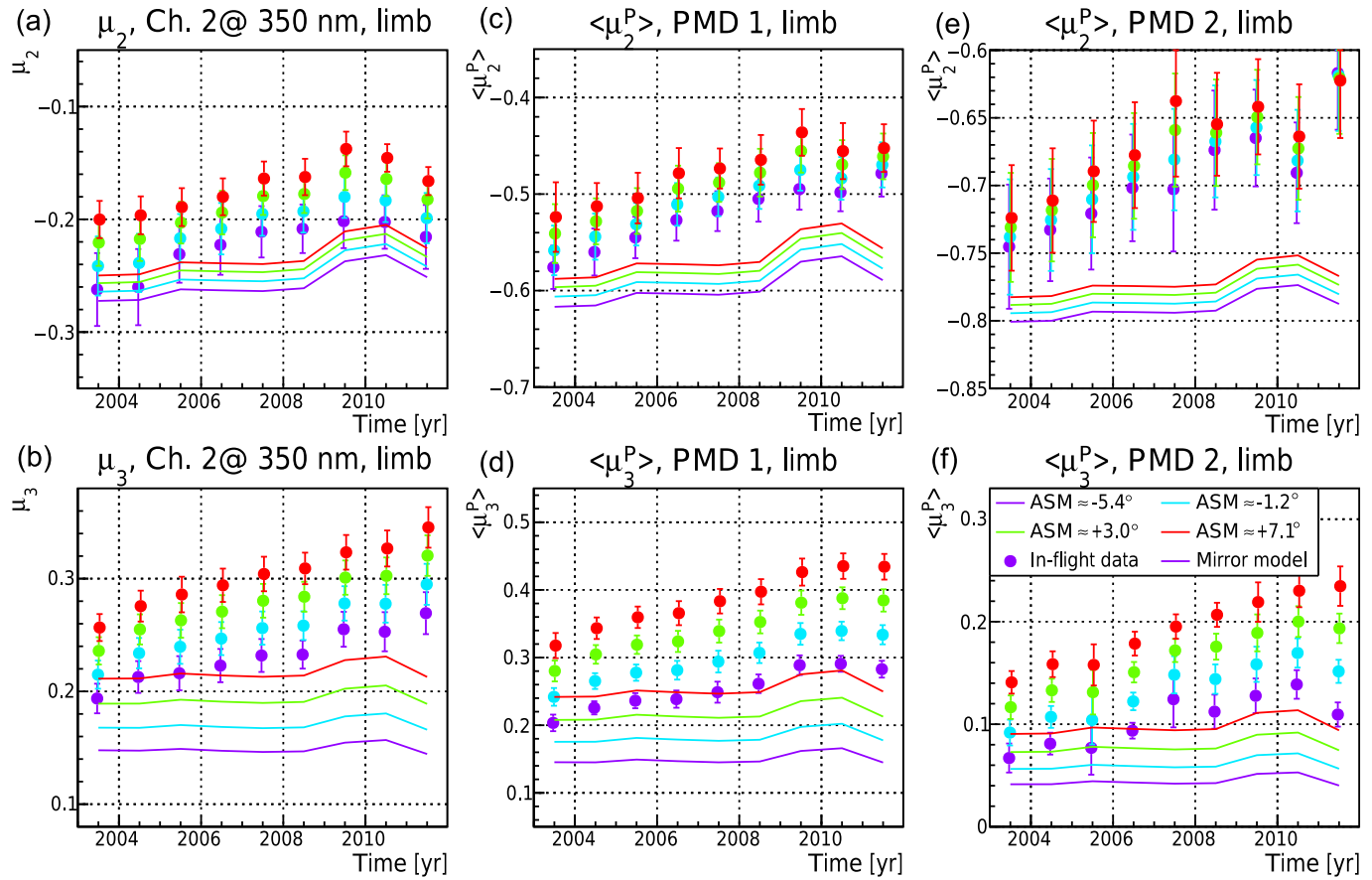

Figure 12. Time dependence of in-flight end-to-end Mueller matrix elements $\mu_{2}$ (a, c, e) and $\mu_{3}(\mathbf{b}, \mathbf{d}, \mathbf{f})$ for limb at $\mathrm{TH} \approx 28 \mathrm{~km}$ for four selected ASM angles from measurements (data points) and the scan mirror model (lines). The error bars include statistical and total systematic errors. (a, b) Channel 2 feature; (c, d) PMD 1; (e, f) PMD 2. The color code signifies the ASM scan angle bin $\left(-5,-1,+3,+7^{\circ}\right)$, increasing on a rainbow color scale. 
tically nonexistent. The reason for this early discrepancy has to lie in the OBM.

\section{Interpretation of results}

In this section, the results for the in-flight MMEs presented above will be interpreted with respect to a change in the OBM polarization sensitivities between the on-ground calibration measurements and in-flight conditions. Already the on-ground calibration revealed an unexpectedly large sensitivity to $45^{\circ}$ polarization end-to-end in nadir, mainly for the PMDs, but also for the science channels. The on-ground calibration campaign was performed for fixed OBM and detector temperatures close to the in-flight temperature settings. But some dedicated measurements were done with varying OBM temperatures, where polarization sensitivities were found to be temperature dependent. The peculiar wavelength and temperature dependence thus pointed to temperature-induced stress birefringence as the likely physical cause (Snel, 1999). The predisperser prism, being the only optical element in the light path of both PMDs and science channels aside from the telescope, would serve as a viable originator of this phenomenon. Even though the prism mount was designed to be stress-free, it may be possible that through the mechanical stress generated by the presence of temperature gradients inside the instrument, birefringence can be generated. It is also possible that a small residual birefringence from the manufacturing process of up to $5 \mathrm{~nm} \mathrm{~cm}^{-1}$ (Keller, 2001) can be further enhanced due to stress. Under the microgravity conditions in-flight, stress may distribute differently, such that the experienced birefringence may change accordingly. Several analyses (Snel, 1999; Slijkhuis and Frerick, 1999) of the on-ground data arrived at different results for the parameters associated with the alleged phase shift, based on different presumptions, definitions and data. Here, we present an analysis of the measured in-flight MMEs as well as the revised on-ground OBM MMEs performed consistently within the frame work of the scan mirror model in order to test the hypothesis of an unintended, stress-induced phase shift inside the predisperser prism.

\subsection{Retarder model}

After the scanner module, the light beam enters the telescope where it undergoes reflections off the two parabolic aluminum mirrors under a small angle and within the same plane. Under these conditions the polarization sensitivity of the telescope can be assumed to be negligible. A possible loss of reflectivity due to degradation would be common to both PMDs and science channels and not be visible in the measured polarization signal. After the telescope, the light enters the predisperser prism, which consists of fused silica glass. It travels for about $1.5 \mathrm{~cm}$ before a part of the beam is internally reflected at an angle close to the Brewster angle $\left(\sim 34^{\circ}\right.$ for fused silica) and emerges nearly $100 \%$ polarized to be directed towards the PMDs after yet another internal reflection, while the major, still partially polarized, part of the beam is transmitted towards the science channels (see Fig. 2 above). Thus, assuming the telescope does not affect the polarized components of the OBM vector, the scan mirror model can be very simply extended by introducing the Mueller matrix for a linear retarder to represent the part of the predisperser prism before the Brewster reflection that can be influenced by birefringence:

$S=I M_{11}^{\mathrm{OBM}} \boldsymbol{\mu}^{\mathrm{OBM}} \mathbf{R}(\delta, \theta) \mathbf{M}(\alpha)(1, q, u, v)^{T}$,

where $\mathbf{R}$ is the Mueller matrix of a linear retarder

$\mathbf{R}(\delta, \theta)=\left(\begin{array}{cccc}1 & 0 & 0 & 0 \\ 0 & c_{2}^{2}+s_{2}^{2} \cos \delta & c_{2} s_{2}(1-\cos \delta) & s_{2} \sin \delta \\ 0 & c_{2} s_{2}(1-\cos \delta) & s_{2}^{2}+c_{2}^{2} \cos \delta & -c_{2} \sin \delta \\ 0 & -s_{2} \sin \delta & c_{2} \sin \delta & \cos \delta\end{array}\right)$

and

$c_{2} \equiv \cos 2 \theta$ and

$s_{2} \equiv \sin 2 \theta$.

Depending on the retarder angle $\theta$, the linear retarder simultaneously rotates the plane of linear polarization and converts linear into circularly polarized light (or vice versa), depending on the retardance $\delta$. At $\theta=0$ or $90^{\circ}$, the crosstalk between $U$ and $V$ is maximized, while at $\theta= \pm 45^{\circ}$ the conversion takes place entirely between $Q$ and $V$. In terms of a birefringent crystal or slab of material, $\theta$ describes the direction of the optic axis, with respect to which the incoming beam is split into an ordinary (polarization direction perpendicular to the optic axis) and extraordinary ray (polarization direction perpendicular to that of the ordinary ray) and $\delta$ is the retardance of the slower ray relative to the faster (typically the ordinary) ray.

The retardance in a slab of thickness $d$ is

$\delta=\frac{2 \pi d}{\lambda}\left(n_{\mathrm{e}}-n_{\mathrm{o}}\right)$.

The actual birefringence is the difference between the indices of refraction for the extraordinary and ordinary ray, $n_{\mathrm{e}}-n_{\mathrm{o}}$. For stress-induced birefringence, this can be related to

$$
\begin{aligned}
B \equiv n_{\mathrm{e}}-n_{\mathrm{o}} & =R(\lambda)\left(\sigma_{1}-\sigma_{2}\right) \\
& =\frac{n(\lambda)^{3}}{2}\left(q_{11}-q_{12}\right)\left(\sigma_{1}-\sigma_{2}\right),
\end{aligned}
$$

where $\sigma_{1}$ and $\sigma_{2}$ are the stresses along the polarization directions of the ordinary and extraordinary rays, and $q_{11}$ and $q_{12}$ are the components of the strain-optic tensor. The refractive index $n$ is wavelength dependent. $R$ is called the stress-optic constant and also depends on wavelength (Sinha, 1978):

$$
R(\lambda)=R\left(\lambda_{0}\right)\left[\frac{n\left(\lambda_{0}\right)}{n(\lambda)}\right]\left[\frac{\lambda^{2}}{\lambda_{0}^{2}}\right]\left[\frac{\lambda_{0}^{2}-\lambda_{1}^{2}}{\lambda^{2}-\lambda_{1}^{2}}\right]\left[\frac{\lambda^{2}-\lambda_{2}^{2}}{\lambda_{0}^{2}-\lambda_{2}^{2}}\right],
$$


with $\quad \lambda_{1}=121.5 \mathrm{~nm}, \quad \lambda_{2}=6900 \mathrm{~nm}$ and $\lambda_{0}$ being a normalization wavelength. For $\lambda_{0}=633 \mathrm{~nm}$, $R=35 \pm 1 \mathrm{nmcm}^{-1} \mathrm{MPa}^{-1} \quad$ (Priestley, 2001). Therefore, it is possible to build a model that determines the retarder matrix for all wavelengths from a measurement at a single wavelength. Here, $\lambda_{0}$ will be set to $300 \mathrm{~nm}$. The retardance at any other wavelength can then be modeled as

$\delta(\lambda)=\delta\left(\lambda_{0}\right) \frac{\lambda_{0}}{\lambda} \frac{R(\lambda)}{R\left(\lambda_{0}\right)}$.

The retarder angle $\theta$ depends on the direction of the applied stress. The type of stress discussed here may be distributed non-uniformly across the prism such that different parts of the beam experience different rotations. Since the relevant phase shift occurs before the Brewster reflection (PMDs) or before the exit from the prism (science channels) and therefore also before wavelength dispersion, the averaged (over the beam cross section) retarder angle can be assumed to be wavelength independent.

\subsection{Fit of retarder parameters}

Based on Eqs. (23) and (22), the vector of retarder parameters $\boldsymbol{\Theta}=\left(\delta\left(\lambda_{0}\right), \theta\right)$ can be found in principle by minimizing the difference between a modeled and measured end-to-end Mueller vector:

$\chi_{\mathrm{N}, \mathrm{L}}^{2}=\left(\boldsymbol{\mu}_{\mathrm{N}, \mathrm{L}}^{\mathrm{Meas}}-\mathbf{X}(\boldsymbol{\Theta})\right)^{T} \cdot \boldsymbol{\Sigma}^{-1} \cdot\left(\boldsymbol{\mu}_{\mathrm{N}, \mathrm{L}}^{\mathrm{Meas}}-\mathbf{X}(\boldsymbol{\Theta})\right)$,

with the instrument model

$\mathbf{X}(\boldsymbol{\Theta}) \equiv \boldsymbol{\mu}_{L, N}(\alpha, \boldsymbol{\Theta})=\boldsymbol{\mu}^{\mathrm{OBM}} \mathbf{R}(\delta, \theta) \mathbf{M}(\alpha)$.

The vector of measurement points $\boldsymbol{\mu}_{\mathrm{N}, \mathrm{L}}^{\text {Meas }}$ contains the measured $\mu_{2}$ and $\mu_{3}$, each for four selected scan angles in nadir and limb, respectively. The covariance matrix $\Sigma$ is determined from the total (statistical and systematic) error as described in Sect. 3.3.2.

\subsubsection{Confidence regions}

Instead of directly combining the nadir and limb data and data for different detectors into one measurement vector, $\chi^{2}$ can be computed for each separately and the resulting distribution as a function of $\left(\delta\left(\lambda_{0}\right), \theta\right)$ can be used to evaluate the validity of the retarder model in terms of its intrinsic consistency. In an ideal case, the best fitting retarder parameters would describe nadir and limb data and each detector equally well. In a statistical sense this means that the regions with

$\Delta \chi^{2}=\chi^{2}(\boldsymbol{\Theta})-\chi_{\text {Min }}^{2}<\Delta \chi_{\text {Max }}^{2}(p)$

for given confidence level $p$ should overlap for individual measurement modes and detectors. The confidence region $\boldsymbol{\Theta}_{p}$ includes all points $\boldsymbol{\Theta}$ with probability $\mathcal{P}\left(\chi^{2}>\right.$ $\left.\chi^{2}(\boldsymbol{\Theta}, v)\right)=1-\operatorname{CDF}\left(\chi^{2}, v\right)>1-p$ for a $\chi^{2}$ distribution with $v$ degrees of freedom with the corresponding cumulative distribution function (CDF). Here $v$ corresponds to the number of considered fit parameters - in this case, $v=2$ (Press et al., 2007). Strictly, this interpretation is only valid if the errors were purely statistical and Gaussian distributed. The systematics-dominated errors of the in-flight data are neither; nevertheless, this approach seems the only practically feasible way to check consistency between independent measurements and combine them in order to maximize information content and to estimate errors on the fit parameters and derived values. This approach is demonstrated in the following.

First, Fig. 13 shows the $\chi^{2}$ distribution for PMD 1, nadir (left panel) and limb (LUT method, right panel) in 2004. The parameter range has been restricted to $|\delta| \leq 45$ and $\theta<90^{\circ}$ due to symmetries in the retarder matrix and because the $\chi^{2}$ for retardances outside the $\pm 45^{\circ}$ limit increases even further.

Inside the considered parameter space, $\chi^{2}$ varies by several orders of magnitude. The plot also shows extended regions of minima located distinctly away from the zero point.

Next, the $99.99 \%$ confidence regions for limb and nadir and different detectors (PMDs 1 and 2, channel 2) are shown in Fig. 14 for each considered year.

In 2003, the confidence regions for all detectors and measurement modes overlap in a common region of $\delta$ between 5 and $10^{\circ}$ and $\theta$ between 10 and $15^{\circ}$. Except for PMD 1, the nadir regions also include a zero retardance, while all limb data are located away from zero. For the following years it can be observed that the limb and nadir regions slowly drift apart almost consistently for all detectors. While for each measurement mode alone there is still an overlap, albeit small, for all detectors, no common overlap exist even for individual detectors after about 2006. There are several possible reasons for this behavior, none of which can be rigorously excluded: the retarder may be an inadequate model to describe the OBM polarization degradation; the mirror degradation parameters may not describe the mirror degradation properly; or an unknown, time-dependent, systematic error impairs the in-flight measurement and its error estimates. The effect of the mirror model degradation parameters is discussed in Sect. 4.3.

Regarding OBM degradation other than the phase shift, it should be considered that, at least for the PMDs, a degradation of optical elements in the light path after the Brewster reflection inside the prism will not depend on the Stokes vector before the prism anymore; therefore, it can affect throughput, but not polarization. This argument holds as long as the prism acts as a near-perfect polarizer. It may be conceivable that some effect, e.g., radiation damage, changes the refractive index of the prism such that the efficiency of the internal Brewster reflection decreases. In such an event, the refractive index change should be noticed elsewhere as well, for instance in the form of a large wavelength shift of the measured spectra. However, such an effect has not been observed. 

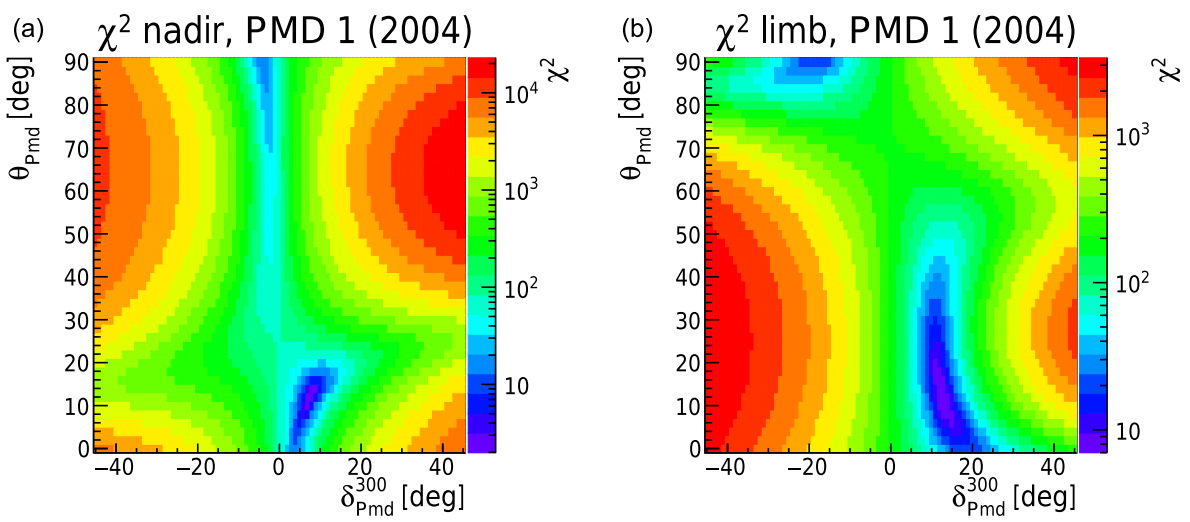

Figure 13. $\chi^{2}$ distribution according to Eq. (30) for PMD 1, nadir (a) and limb (b) in 2004. The retardance parameter is on the $x$ axis and the retarder angle on the $y$ axis; the color code depicts $\chi^{2}$ on a logarithmic (rainbow) color scale.

\subsubsection{Determination of model parameters and errors}

Especially in limb, it is important to have a time-dependent in-flight correction to the mirror model MMEs for all wavelengths and PMDs and science channels alike. That is why, even though the observed drift of limb and nadir data does not raise too much confidence in the hypothesis of a timedependent OBM phase shift, the retarder model is applied to determine the most likely retarder parameters and their errors as a function of time. This is done by the process of marginalizing the respective other parameter, i.e., by integrating each retarder parameter weighted with the likelihood derived from the CDF of the $\chi^{2}$ distribution (Verde, 2010). The likelihood for any combination of measurement modes and detectors can be obtained from the product of the respective, normalized individual distributions:

$\mathcal{L}^{C}\left(\boldsymbol{\Theta}^{\mathrm{Fit}}\right)=\prod_{i} \frac{\mathcal{P}^{i}(\boldsymbol{\Theta})}{\int \mathcal{P}^{i}(\boldsymbol{\Theta}) \mathrm{d} \boldsymbol{\Theta}}$

The mean values of a generic fit parameter or a derived parameter $\Theta$ (such as the retarder-corrected in-flight MMEs) and their errors can be computed from this distribution as follows:

$$
\begin{aligned}
\left\langle\Theta^{C}\right\rangle & =\int \Theta^{C} \mathcal{L}^{C}(\Theta) \mathrm{d} \Theta, \\
\sigma^{2}\left(\Theta^{C}\right) & =\int\left(\Theta-\left\langle\Theta^{C}\right\rangle\right)^{2} \mathcal{L}^{C}(\Theta) \mathrm{d} \Theta,
\end{aligned}
$$

for all considered combinations $C$.

After deriving the retarder parameters in the initial analysis step, an iteration of the entire chain of fits has to be performed. The reflectances and polarization signals are corrected for the polarization of the science channels with the MMEs computed with the retarder parameters from the initial step, using Eq. (16) with the updated MMEs. The effect of this iteration on the value for the retardance is almost negligible, but the retarder angle is shifted down by about $3^{\circ}$.

\subsubsection{Results}

Figure 15 shows the results for the retarder parameters for different observation modes and detector combinations after the iteration step.

It demonstrates how adding information from different detectors, e.g., PMD 1 and channel 2, or combining limb and nadir, subsequently changes retarder values and reduces errors. For instance, in nadir there is not much information on the retarder angle because for small retardances $\chi^{2}$ (or likewise the probability) does not depend on the retarder angle. In limb, larger retardances are favored and the retarder angle is therefore constrained better. The evident offset between channel 2 and PMD 1 comes from the peculiar confidence region of channel 2 which includes large values of $\theta>45^{\circ}$ and negative retardances. Only the combination with the PMDs restricts this region to $\theta<30^{\circ}$ and positive retardances. The plot also shows how the combination of limb and nadir data results in a compromise which is dominated by the information contained in the limb data. For PMD 2, the sensitivity to the phase shift is considerably smaller than for PMD 1 and channel 2 due to its wavelength dependence, while the sensitivity to errors in the calibration is larger, as can be seen for instance in the error bars of Fig. 11. Therefore, the final retarder values to be used for polarization determination and correction will eventually be derived from a combination of limb and nadir, PMD 1 and channel 2. The values shown in Fig. 15 were obtained with the LUT method for the PMDs in limb and the extrapolation method otherwise. Retarder parameters obtained by using the extrapolation method in limb entirely differ by about 1 to maximum $2^{\circ}$ for the final result. Parameters change within a similar margin when the off-diagonal elements in the systematic covariance matrix are set to zero.

The end-to-end MMEs calculated from the retarder model with the fitted parameters are shown in Figs. 16 and 17 together with the MMEs calculated from the original mirror model and the in-flight data. The errors on the end-to-end 


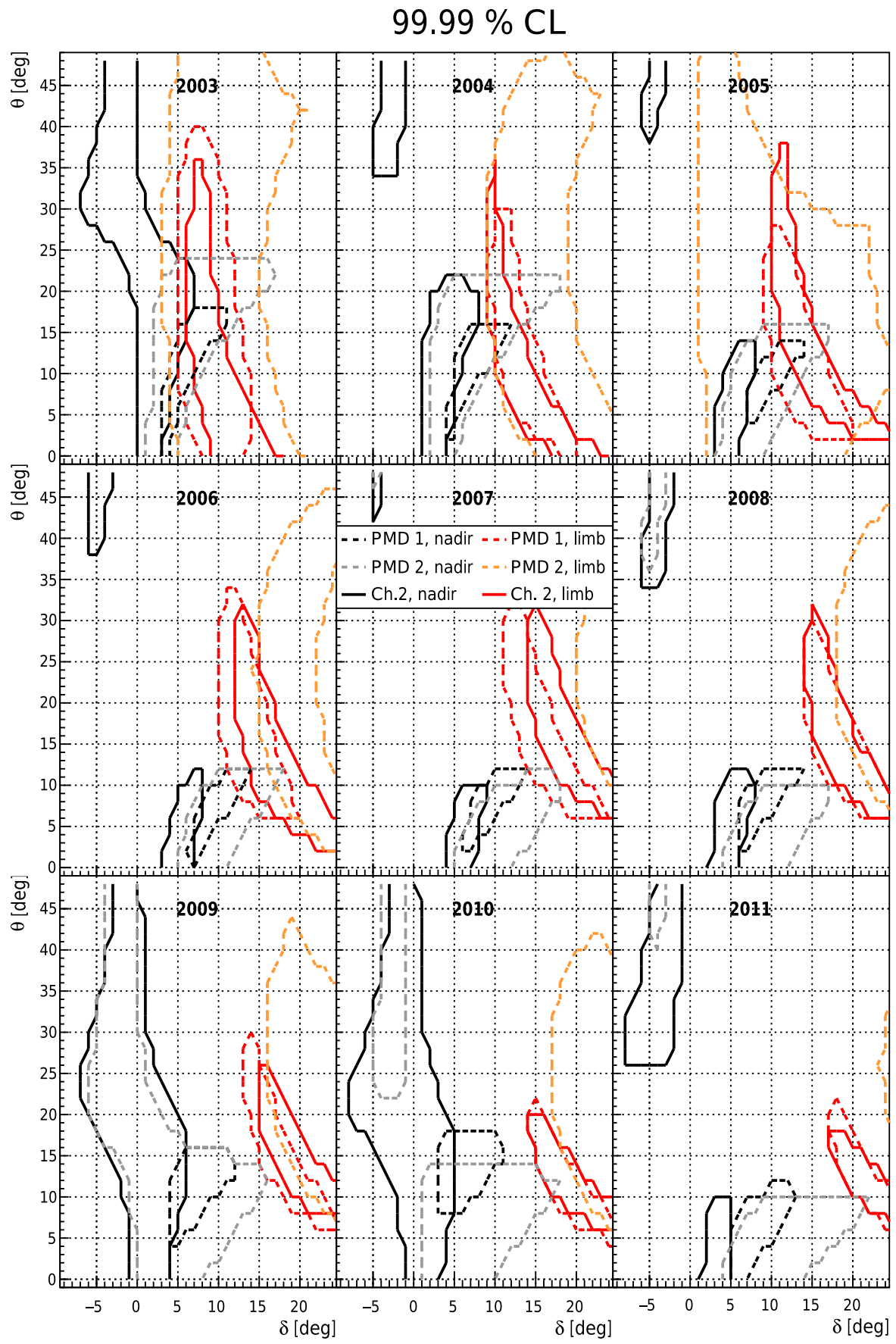

Figure 14. The $99.99 \%$ confidence region contours for limb and nadir, PMDs 1 and 2, and channel 2 for each year starting from 2003 (top left) to 2011 (bottom right).

MMEs (Eq. 34) are typically less than a few $10^{-3}$ and therefore much smaller than the error on the data, which is why they are not shown on the plot.

The retarder model seems to fix the largest differences in nadir and mostly also in limb. An issue remains with the time dependence in limb, especially towards the later part of the mission. The main effect of the retarder model applied to the data will be an improvement of the polarization determination, reducing systematic errors in the limb UV down to about $10 \%$ and therefore by a factor of about 2 . The polarization correction in the UV in channel 1 and for channel 2 around $350 \mathrm{~nm}$ will also considerably improve. However, the calibration for the unpolarized response will be nearly unaffected. 
(a)

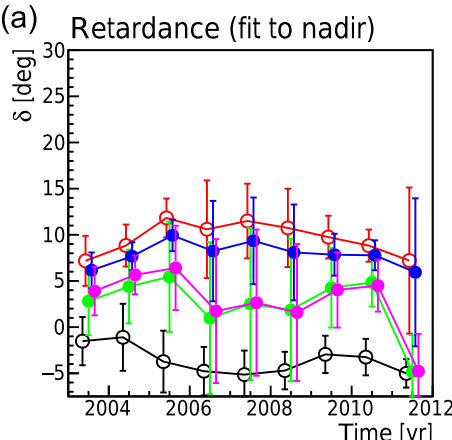

(d)

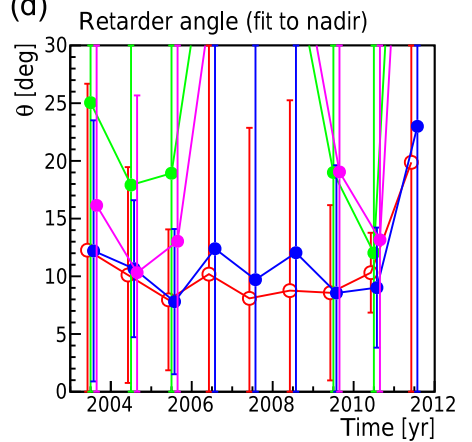

(b)

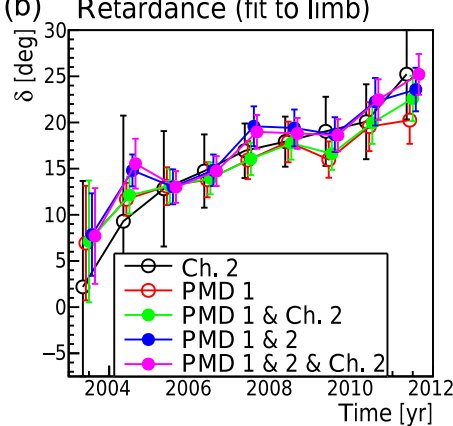

(e) Retarder angle (fit to limb)

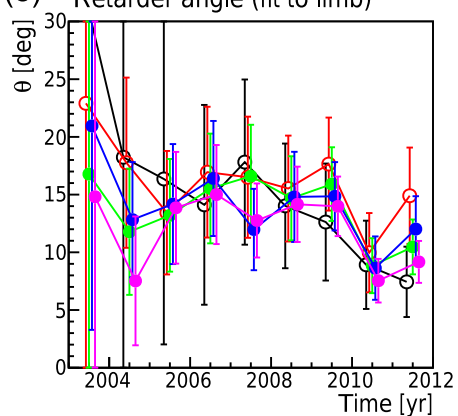

(c)

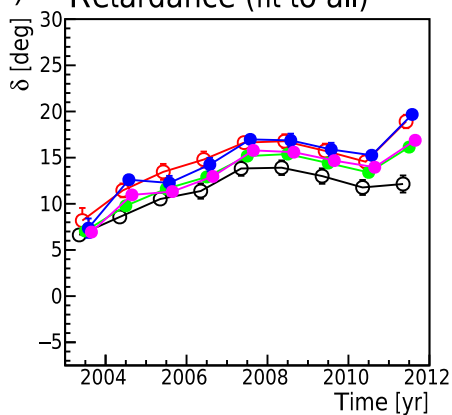

(f)

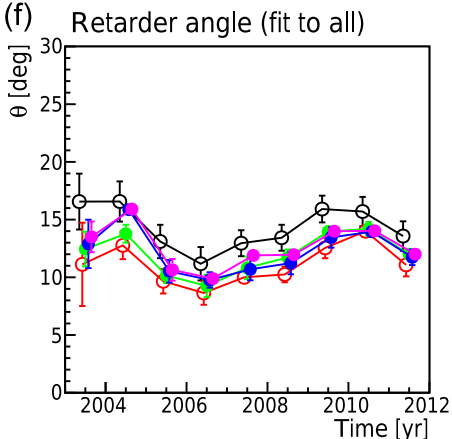

Figure 15. Fit results for the parameters $\delta(\mathbf{a}, \mathbf{b}, \mathbf{c})$ and $\theta(\mathbf{d}, \mathbf{e}, \mathbf{f})$ vs. time using only nadir (a, d), only limb (LUT method for the PMDs, b, e), or limb and nadir combined (c, f). The different colors are for different combinations of detectors.
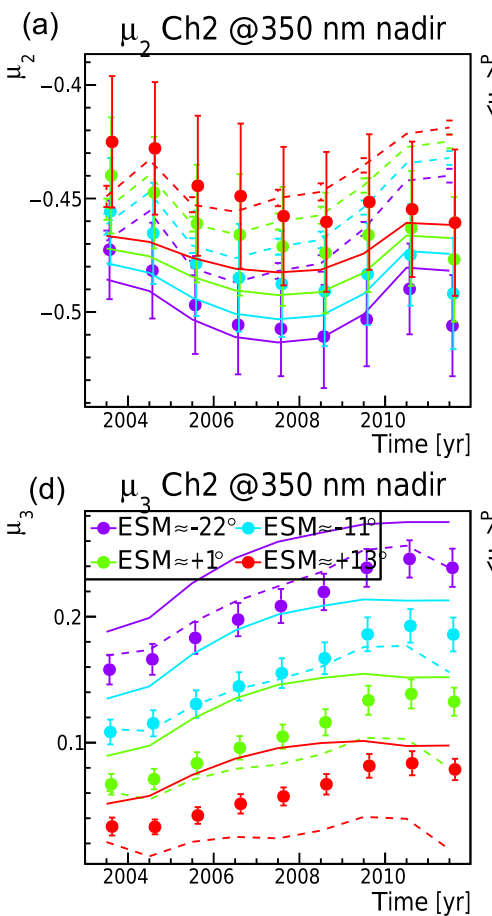

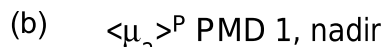

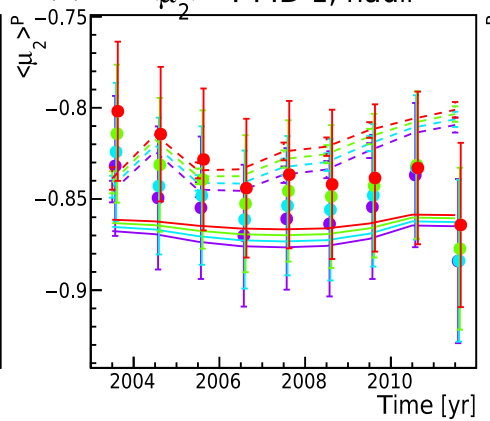

(e) $\quad<\mu_{3}>$ P PMD 1, nadir

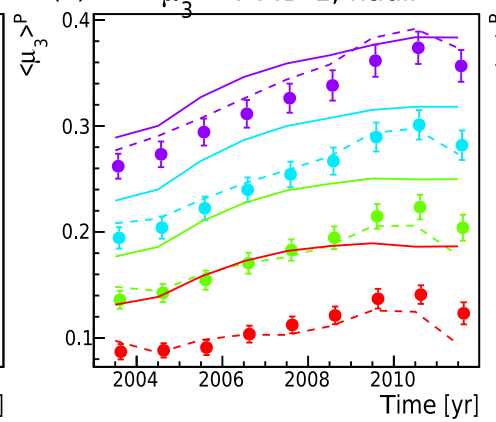

(c)

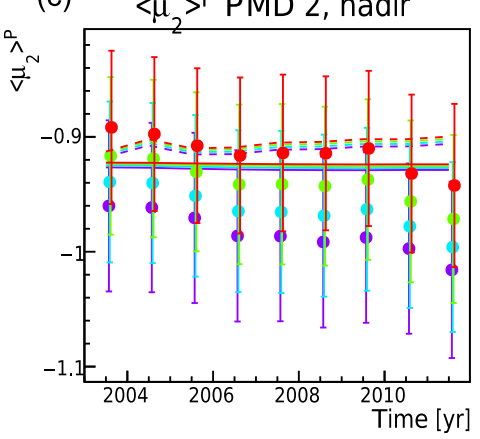

(f)

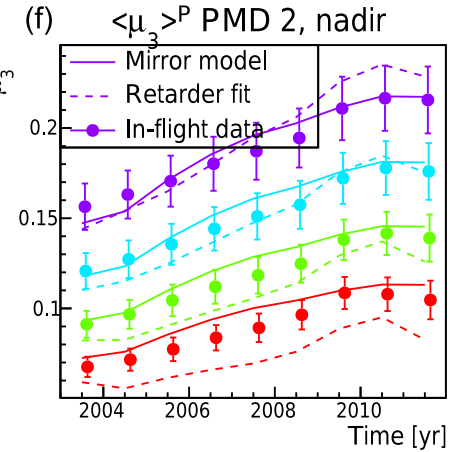

Figure 16. Time dependence of in-flight end-to-end Mueller matrix elements $\mu_{2}(\mathbf{a}, \mathbf{b}, \mathbf{c})$ and $\mu_{3}$ (d, e, f) for nadir for four selected ESM angles from measurements (data points), the scan mirror model without the retarder (solid lines) and the mirror model with the retarder (dashed lines). The error bars of the data include statistical and total systematic errors. (a, d) Channel 2 feature; (b, e) PMD 1; (c, f) PMD 2.The color code signifies the ESM scan angle bin $\left(-22,-11,+1,+13^{\circ}\right)$, increasing on a rainbow color scale. 


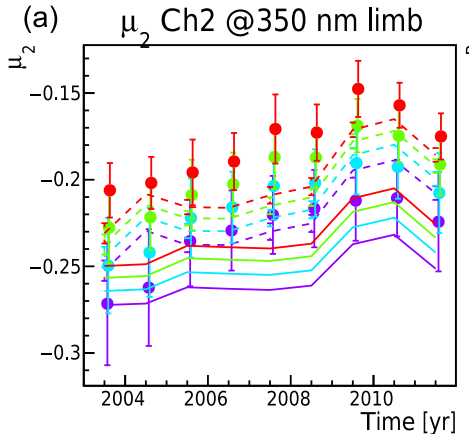

(d)

$\mu_{3}$ Ch2 @350 nm limb

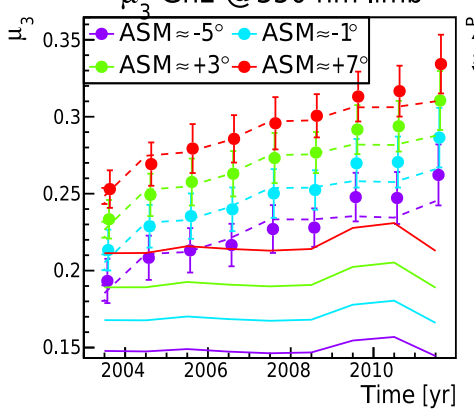

(b) $<\mu_{2}>^{P}$ PMD 1, limb

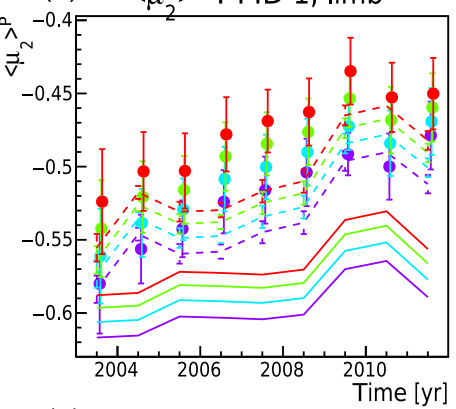

(e)

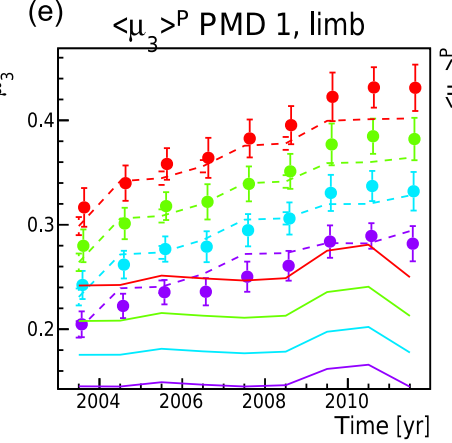

(c)

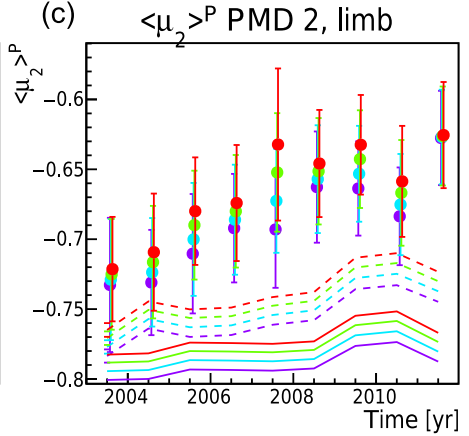

(f)

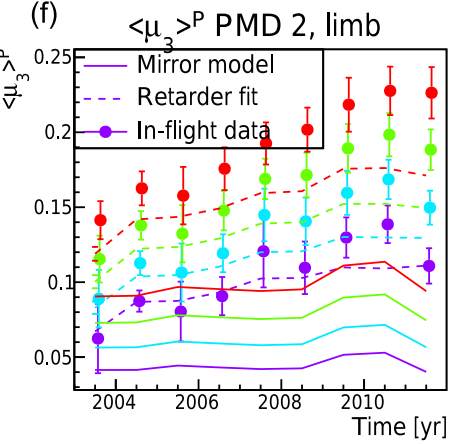

Figure 17. Time dependence of in-flight end-to-end Mueller matrix elements $\mu_{2}(\mathbf{a}, \mathbf{b}, \mathbf{c})$ and $\mu_{3}(\mathbf{d}, \mathbf{e}, \mathbf{f})$ for limb at $\mathrm{TH} \approx 28 \mathrm{~km}$ for four selected ASM angles from measurements (data points), the scan mirror model without the retarder (solid lines) and the mirror model with the retarder (dashed lines). The error bars include statistical and total systematic errors. (a, d) Channel 2 feature; (b, e) PMD 1; (c, f) PMD 2. The color code signifies the ASM scan angle bin $\left(-5,-1,+3,+7^{\circ}\right)$, increasing on a rainbow color scale.

\subsection{Dependence of retarder parameters on the scan mirror model}

The fit procedure described in Sect. 4.2 can be applied in an analogous way to fit parameters of the mirror degradation, i.e., thicknesses on ESM and ASM mirrors and the refractive index at 350 (using PMD 1 and channel 2) and $480 \mathrm{~nm}$ (using PMD 2). The measurement vectors used are the same as for the retarder fit. The combination of different detectors and observation modes is more complex in such a fit because of the relationship of the fit parameters to certain subsets of data. The fit aims at constraining these six independent parameters from selected detector and observation mode combinations which maximize the information content. The investigated range was from 0 to $100 \mathrm{~nm}$ in contaminant thicknesses, from 1 to 1.5 for the real part and from -0.5 to 0 in the imaginary part of the refractive index.

Together with the unpolarized response constrained to lie within limits consistent with the solar calibration data, the allowed parameter range can be considerably reduced. Fit parameters, for instance thickness and refractive index, tend to be highly anticorrelated. This can be explained by the equations describing the effect of a thin layer such as the contaminant on the mirror substrate, where the relevant term for the reflection coefficient enters as a product of layer thickness and refractive index (Krijger et al., 2014). This high correlation inhibits the unambiguous determination of all parame- ters, but a few qualitative statements can be made based on this investigation.

First, within the scanned parameter range, it is not possible to find a physically reasonable set of parameters that could describe the measured in-flight MMEs without an additional phase shift introduced by the retarder. This finding proves that there is indeed an on-ground to in-flight change inside the OBM. Second, it is possible to find physically reasonable sets of parameters that fit the data when fixing the retarder parameters to those obtained for 2003. That means that a single on-ground to in-flight change in the OBM would suffice to describe the measurements at all times in combination with mirror model parameters that tend to be different from the ones derived from unpolarized calibration data (see Fig. 10). In particular, the range of possible combinations of thickness and refractive index is inconsistent with those values. In fact, applying a time-dependent refractive index with a constant phase shift consistently leads to better agreement between the model and data than a time-dependent phase shift with a constant refractive index.

At the very least this means that the thicker the contamination layer is, the more the retarder parameters depend on the mirror model parameters. Furthermore, the findings suggest that the inclusion of polarization data into the fits of the mirror degradation parameters may improve or even resolve the degeneracy between different fit parameters. 


\subsection{OBM vector fits}

Nadir and limb data can also be combined to fit the three polarization components of the OBM vector directly for each detector separately. The fit model $\mathbf{X}(\boldsymbol{\Theta})$ (Eq. 30) is linear in this case, with its independent parameters consisting only of the components of the OBM Stokes vector. In contrast to the retarder model, which preserves polarization, in this fit the total OBM polarization, $p=\sqrt{\mu_{2}^{2}+\mu_{3}^{2}+\mu_{4}^{2}}$, can change. The only constraint imposed is that it stays within physical limits, i.e., $p \leq 1$.

The results of this test show that the fitted OBM vector $\boldsymbol{\mu}_{\mathrm{OBM}}^{\mathrm{Fit}}$ is consistent with the phase-shifted on-ground vector when scaled with a factor accounting for the change in polarization, i.e.,

$\boldsymbol{\mu}_{\mathrm{OBM}}^{\mathrm{Fit}} \approx \boldsymbol{\mu}_{\mathrm{OBM}} \mathbf{R}\left(\delta^{\mathrm{Fit}}, \theta^{\mathrm{Fit}}\right) \frac{p^{\mathrm{Fit}}}{p^{\mathrm{OBM}}}$,

where $\left(\delta^{\mathrm{Fit}}, \theta^{\mathrm{Fit}}\right)$ are the retarder fit parameters from Sect. 4.2.3. This holds in particular for PMDs 1 and 2, which is remarkable because it also proves consistency with the wavelength dependence ascribed to the stress-induced retardance (Eq. 29). The polarization scale factor only differs from 1 by at most $2 \%$. Also for this fit, a considerable dependence on the mirror model parameters was found.

\subsection{On-ground polarization phase shift}

Finally, the use of the combined scan mirror and retarder model enables the interpretation of the on-ground measurements in terms of an on-ground OBM phase shift, which is analogous to the analyses of Snel (1999) and Slijkhuis and Frerick (1999). This analysis is based on the presumption that, by original design, the PMDs should have almost $100 \%$ sensitivity to perpendicular polarized light entering the OBM and no sensitivity to $45^{\circ}$ or circular polarization. This means that

$\boldsymbol{\mu}_{\mathrm{OBM}}^{\mathrm{on}-\mathrm{grd}} \equiv\left(1,-p^{\mathrm{OBM}}, 0,0\right)$

allowing for a non-perfect polarizer with $p^{\mathrm{OBM}} \leq 1$. By inverting

$\boldsymbol{\mu}_{\mathrm{OBM}}=\boldsymbol{\mu}_{\mathrm{OBM}}^{\text {on-grd }} \mathbf{R}\left(\delta^{\text {on-grd }}, \theta^{\text {on-grd }}\right)$,

one can find a set of parameters ( $\left.\delta^{\text {on-grd }}, \theta^{\text {on-grd }}\right)$ that matches the measured on-ground OBM vector $\boldsymbol{\mu}_{\mathrm{OBM}}$ within errors. Likewise, by replacing $\mu_{\mathrm{OBM}}$ with the result of the in-flight fit $\boldsymbol{\mu}_{\mathrm{OBM}}^{\mathrm{Fit}}$ (Eq. 36), another set ( $\left.\delta^{\text {in-flight }}, \theta^{\text {in-flight }}\right)$ can be found that relates the non-phase-shifted on-ground vector $\boldsymbol{\mu}_{\mathrm{OBM}}^{\text {on-grd }}$ directly to the fitted in-flight vector and thus puts the two parameter sets $\left(\delta^{\text {on-grd }}, \theta^{\text {on-grd }}\right)$ and $\left(\delta^{\text {in-flight }}, \theta^{\text {in-flight }}\right)$ in perspective. The (approximate) results of this exercise are

$$
\begin{aligned}
\delta^{\text {on-grd }} & =35.5^{\circ} \pm 0.5^{\circ}, \quad \theta^{\text {on-grd }}=45.0^{\circ} \pm 2.0^{\circ}, \\
\delta^{\text {in-flight }} & =42.0^{\circ} \pm 1.5^{\circ}, \quad \theta^{\text {in-flight }}=35.0^{\circ} \pm 1.0^{\circ} .
\end{aligned}
$$

Interpreting the on-ground phase shift in terms of stress birefringence $B$ using Eq. (26) leads to

$B \equiv\left(n_{\mathrm{e}}-n_{\mathrm{o}}\right)^{\text {on-grd }}=2 \cdot 10^{-6}=20 \mathrm{~nm} \cdot \mathrm{cm}^{-1}$,

which is a value at least 4 times as high as what could be expected from residual birefringence of the material (Keller, 2001). Attributing the entire birefringence to stress, using $R\left(\lambda_{0}=633 \mathrm{~nm}\right)=35 \mathrm{~nm} \cdot \mathrm{cm}^{-1} \cdot \mathrm{MPa}^{-1}$ (Priestley, 2001) and Eq. (28), an estimate for the on-ground stress amounts to

$$
\begin{aligned}
\Delta \sigma & =\sigma_{1}-\sigma_{2}=\frac{n_{\mathrm{e}}-n_{\mathrm{o}}}{R(\lambda=300 \mathrm{~nm})} \\
& \approx \frac{2 \cdot 10^{-6}}{39.8 \cdot 10^{-7}} \mathrm{MPa}=0.5 \mathrm{MPa},
\end{aligned}
$$

which seems to be quite large considering that the prism mount was originally designed to be stress-free. The source of the stress causing the observed birefringence was never found, despite careful investigations of the instrument during on-ground testing. Both a bread board model and the proto-flight model of the instrument showed no abnormal polarization effects at room temperature and a linear buildup of stress birefringence as temperature was lowered to operational conditions (Snel, 1999). The effect could be reproduced by physically stressing the predisperser prism on the component level, but the stress-free mount of the prism prevented this from happening when prism and mount were tested together.

Given the very small thermal expansion coefficient of fused silica and the low intrinsic birefringence, the observed large sensitivity to temperature of the order of $1^{\circ} \mathrm{K}^{-1}$ is unlikely to be generated inside the prism itself but instead possibly inside the instrument or even in the prism mount. Another curious feature is the on-ground retarder angle, which happens to be the singular angle that ensures that $\mu_{3} \equiv 0$ over the entire wavelength range and all retardances. It seems a bit peculiar that an unintended stress should select exactly this particular direction.

The in-flight phase shift change on the other hand seems to be relatively small compared to the already large on-ground phase shift and amounts to an approximately $15 \%$ difference in stress, with a $10^{\circ}$ rotation of stress angle. Since the OBM temperature was stable in-flight and did not change with respect to on-ground calibration measurements, the difference in gravitational force has always been suspected to cause the in-flight change. On the other hand it was also discussed that the entrance window into the vacuum chamber used during the on-ground campaign may at least be responsible for part of the observed phase shift. If that were the case, the onground to in-flight change may be simply due to the absence 
of this so-called "OPTEC window" in flight. With the currently available data, it is not possible to clearly identify the reason for the observed behavior on the ground and in flight. Further analysis of all available calibration data may be necessary.

\section{Conclusions}

A novel statistical approach for the in-flight polarization calibration of the SCIAMACHY PMDs and a part of its science channels is presented. It exploits the relationship between polarization and measured reflectance. This approach can in principle be further refined and adapted for the polarization calibration of other instruments which measure polarization, such as GOME, GOME-2 or even POLDER/PARASOL. The overarching requirements for the polarization sensitivity measurement are a sufficiently accurate model or data applicable to the particular measurement conditions or sufficient statistics and leverage to extrapolate to a limiting model to determine polarization signals. The resulting polarization signals should cover a significant portion of the $(q, u)$ plane to provide enough leverage for the determination of polarization sensitivities.

The general applicability to other instruments has not been discussed in much detail here because it depends on the specific instrument, its measurement modes and available data. Limiting models can be in principle improved by including information on known wind speeds or surface reflectance. An extension of well-established calibration methods employing natural targets (see Frouin, 2013, for an overview) with the extrapolation method to include polarization may also be worth considering. For SCIAMACHY, however, using a few selected oceanic sites resulted in too-small leverage in $(q, u)$ to retrieve meaningful information; for other instruments such as PARASOL or MODIS with many observations of the same scene from different angles, this may not pose a problem. For MODIS, for instance, cross calibration with SeaWiFS data and modeled polarization values has been successfully applied (Meister et al., 2009). The modeled relationship between reflectance and polarization may be replaced by suitable data, such as accurately calibrated data from the POLDER or PARASOL instruments. Co-located reflectance measurements may provide a reference reflectance to determine polarization signals when dedicated polarization measurements are not available. For instance, the combination of MERIS reflectance data co-located with SCIAMACHY measurements, together with a statistical distribution of $(q, u)$ vs. reflectance derived from PARASOL data matched with SCIAMACHY viewing geometries, resulted in an independent measurement of science channel polarization signals at MERIS wavelengths, e.g., 510, 665 and $885 \mathrm{~nm}$. The derived polarization sensitivities for nadir were roughly consistent with expectation (Liebing et al., 2014). The investigated time span was only 1 month, August 2007, such that any further interpretation of the results was inhibited by toolarge uncertainties.

The results presented here are based on the polarizationreflectance relationship inferred directly from RTM simulations or extrapolated to a limiting case. For wavelengths below $\sim 500 \mathrm{~nm}$ they prove to be stable enough for further investigation of the instrument degradation. First, it was found that the instrument model used to calibrate the scan-angledependent degradation of SCIAMACHY due to contamination of its scan mirrors neither reproduces the in-flight polarization sensitivities in these channels at the beginning of the mission nor their time dependence. The effect is largest for the limb data. Second, the observed discrepancies between the model and data in the early part of the mission could be clearly traced back to an on-ground to in-flight change in the polarization sensitivity inside the optical bench of the instrument. The values are consistent with an extended instrument model that includes both the mirror degradation and stress-induced birefringence inside the predisperser prism. The model was used to fit the parameters of a linear retarder that generates a polarization phase shift.

Several tests were performed to investigate the reason for the observed phase shift, both on the ground and in flight, as well as its time dependence. A retrospect analysis of the on-ground calibration data shows that an unexplained, large phase shift was already present at that time and that the difference between the on-ground and in-flight phase shifts is comparatively small. The time dependence may indeed be an artifact of the scan mirror model, resulting from the imposed assumptions on the properties of the mirror contaminant. Since the polarization data show a sizeable sensitivity to these properties, the inclusion of these data in addition to the solar measurements in the calibration approach of the scan mirror model may actually help to disentangle some of the ambiguities between different degradation parameters.

Even though the search for the origin of the phase shift remained inconclusive, the results of this investigation considerably improve the polarization calibration of the measured SCIAMACHY radiances, in particular in limb. As an added benefit, the new calibration, together with an improved algorithm applied in version 9 of the operational level 0 to 1 processor, enables the determination of the atmospheric polarization, i.e., Stokes $q$ for all PMD wavelengths and $u$ at $850 \mathrm{~nm}$, with a relative accuracy on the order of $10 \%$. Although this is far from the accuracies achieved by dedicated instruments such as POLDER, the new SCIAMACHY polarization data may provide valuable additional information - on a statistical basis - on, for example, cloud or aerosol properties for suitable observing geometries.

Data availability. This analysis was performed using operational level 1B SCIAMACHY data (version 8), accessible via https://earth.esa.int/web/guest/-/sciamachy-localized-atmosphericspectra-in-the-uvvisnear-ir. 
Competing interests. The authors declare that they have no conflict of interest.

Acknowledgements. SCIAMACHY is a national contribution to the ESA Envisat project, funded by Germany, the Netherlands and Belgium. SCIAMACHY data have been provided by ESA. This work has been funded by the German Aerospace Center (DLR), the SCIAMACHY Quality Working Group project (ESA) and by the University of Bremen.

The article processing charges for this open-access publication were covered by the University of Bremen.

Edited by: Udo Friess

Reviewed by: Ruediger Lang and one anonymous referee

\section{References}

Bovensmann, H., Burrows, J. P., Buchwitz, M., Frerick, J., Noël, S., Rozanov, V. V., Chance, K. V., and Goede, A. H. P.: SCIAMACHY - Mission Objectives and Measurement Modes, J. Atmos. Sci., 56, 127-150, 1999.

Cox, C. and Munk, W.: Measurement of the Roughness of the Sea Surface from Photographs of the Sun's Glitter, J. Opt. Soc. Am., 44, 838-850, https://doi.org/10.1364/JOSA.44.000838, 1954.

Emde, C. and Mayer, B.: Simulation of solar radiation during a total eclipse: a challenge for radiative transfer, Atmos. Chem. Phys., 7, 2259-2270, https://doi.org/10.5194/acp-7-2259-2007, 2007.

Frouin, R. (Ed.): In-flight Calibration of Satellite Ocean-Colour Sensors, Vol. No. 14 of Reports of the International Ocean Colour Coordinating Group, IOCCG, Dartmouth, Canada, available at: http://www.ioccg.org/reports/IOCCG_Report_14_2013. pdf (last access: 7 January 2018), 2013.

Gottwald, M. and Bovensmann, H. (Eds.): SCIAMACHY - Exploring the Changing Earth's Atmosphere, Springer, Dordrecht Heidelberg London New York, https://doi.org/10.1007/978-90-4819896-2, 2011.

Keller, C. U.: Instrumentation for Astrophysical Spectropolarimetry, in: Astrophysical Spectropolarimetry, edited by: Trujillo-Bueno, J., Moreno-Insertis, F., and Sanchez, F., Cambridge University Press, Cambridge, 303-354, https://doi.org/10.1017/CBO9780511564802.012, 2001.

Kokhanovsky, A. A., Budak, V. P., Cornet, C., Duan, M., Emde, C., Katsev, I. L., Klyukov, D. A., Korkin, S. V., C-Labonnote, L., Mayer, B., Min, Q., Nakajima, T., Ota, Y., Prikhach, A. S., Rozanov, V. V., Yokota, T., and Zege, E. P.: Benchmark results in vector atmospheric radiative transfer, J. Quant. Spectrosc. Ra., 111, 1931-1946, https://doi.org/10.1016/j.jqsrt.2010.03.005, 2010.

Krijger, J. M.: SCIAMACHY On-Ground Polarisation Recalibration, SRON-SQWG3_TN-2017-001, SRON, 2017.

Krijger, J. M., Tanzi, C. P., Aben, I., and Paul, F.: Validation of GOME polarization measurements by method of limiting atmospheres, J. Geophys. Res., 110, D07305, https://doi.org/10.1029/2004JD005184, 2004.

Krijger, J. M., Snel, R., van Harten, G., Rietjens, J. H. H., and Aben, I.: Mirror contamination in space I: mirror modelling, Atmos.
Meas. Tech., 7, 3387-3398, https://doi.org/10.5194/amt-7-33872014, 2014.

Liebing, P., Bramstedt, K., Noël, S., Rozanov, V., Bovensmann, H., and Burrows, J. P.: Polarization data from SCIAMACHY limb backscatter observations compared to vector radiative transfer model simulations, Atmos. Meas. Tech., 6, 1503-1520, https://doi.org/10.5194/amt-6-1503-2013, 2013.

Liebing, P., Snel, R., Bramstedt, K., and Krijger, M.: An Assessment of the In-flight Polarization Response of SCIAMACHY, AGU Fall Meeting Abstracts, available at: http://www.iup. uni-bremen.de/sciamachy/polarisation/AGU/SciaPol_pix.pdf (last access: 7 January 2018), 2014.

Maystre, D.: Theory of Wood's Anomalies, in: Plasmonics: From Basics to Advanced Topics (Springer Series in Optical Sciences), Springer, 2012.

McLinden, C. A., Haley, C. S., Lloyd, N. D., Hendrick, F., Rozanov, A., Sinnhuber, B.-M., Goutail, F., Degenstein, D. A., Llewellyn, E. J., Sioris, C. E., Roozendael, M. V., Pommereau, J. P., Lotz, W., and Burrows, J. P.: Odin/OSIRIS observations of stratospheric BrO: Retrieval methodology, climatology, and inferred $\mathrm{Br}_{y}$, J. Geophys. Res., 115, D15308, https://doi.org/10.1029/2009JD012488, 2010.

Meister, G., Franz, B. A., Kwiatkowska, E. J., Eplee, R. E., and McClain, C. R.: Detector dependency of MODIS polarization sensitivity derived from on-orbit characterization, Proc. SPIE, 7452, 7452, https://doi.org/10.1117/12.825385, 2009.

Press, W. H., Teukolsky, S. A., Vetterling, W. T., and Flannery, B. P.: Numerical Recipes 3rd Edn.: The Art of Scientific Computing, Cambridge University Press, New York, NY, USA, 3 Edn., 2007.

Priestley, R.: Birefringence dispersion in fused silica for DUV lithography, Proc. SPIE, 4346, 1300-1305, https://doi.org/10.1117/12.435666, 2001.

Rozanov, V., Rozanov, A., Kokhanovsky, A., and Burrows, J.: Radiative transfer through terrestrial atmosphere and ocean: Software package \{SCIATRAN\}, J. Quant. Spectrosc. Ra., 133, 1371, https://doi.org/10.1016/j.jqsrt.2013.07.004, 2014.

Rozanov, V. V. and Rozanov, A. V.: Software Package SCIATRAN (Radiative Transfer Model and Retrieval Algorithm), IUP, available at: http://www.iup.uni-bremen.de/sciatran/free_downloads/ users_guide_sciatran.pdf (last access: 7 January 2018), 2016.

Sinha, N. K.: Normalised dispersion of birefringence of quartz and stress optical coefficient of fused silica and plate glass, Phys. Chem. Glasses, 19, 69-77, 1978.

Sinnhuber, B.-M., Sheode, N., Sinnhuber, M., Chipperfield, M. P., and Feng, W.: The contribution of anthropogenic bromine emissions to past stratospheric ozone trends: a modelling study, Atmos. Chem. Phys., 9, 2863-2871, https://doi.org/10.5194/acp-92863-2009, 2009.

Slijkhuis, S. and Frerick, J.: SCIAMACHY Polarisation Sensitivity, in: ERS-ENVISAT Symposium "Looking down to Earth in the New Millennium", 461, p. 1, 1999.

Snel, R.: SCIAMACHY on-ground performance: polarisation phase shift, in: Proceedings of ESAMS'99 - European Symposium on Atmospheric Measurements from Space, ESTEC, Nordwijk, The netherlands, 18-22 January 1999 (WPP 161, Vol. 2), ESA, Nordwijk, Netherlands, 725-729, 1999. 
Tilstra, L. G. and Stammes, P.: Earth reflectance and polarization intercomparison between SCIAMACHY onboard Envisat and POLDER onboard ADEOS-2, J. Geophys. Res., 112, D11304, https://doi.org/10.1029/2006JD007713, 2007.

Tilstra, L. G. and Stammes, P.: SCIAMACHY Level-1 V7.03: Earth Reflectance and Polarisation, KNMI-RP-2010-01, KNMI, 2010.

Verde, L.: Statistical Methods in Cosmology, in: Lecture Notes in Physics, Berlin Springer Verlag, edited by: Wolschin, G., Vol. 800 of Lecture Notes in Physics, Berlin Springer Verlag, 147177, https://doi.org/10.1007/978-3-642-10598-2_4, 2010. von Savigny, C., Ulasi, E. P., Eichmann, K.-U., Bovensmann, H., and Burrows, J. P.: Detection and mapping of polar stratospheric clouds using limb scattering observations, Atmos. Chem. Phys., 5, 3071-3079, https://doi.org/10.5194/acp-5-3071-2005, 2005. 